Environnement urbain

Urban Environment

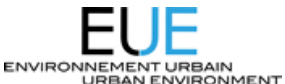

\title{
L'impact des migrations internationales et des mobilités résidentielles sur l'évolution socio-spatiale des agglomérations de Luxembourg et Bruxelles
}

\section{Sébastien Lord, Tim Cassiers et Philippe Gerber}

Volume 8, 2014

URI : https://id.erudit.org/iderudit/1027743ar

DOI : https://doi.org/10.7202/1027743ar

Aller au sommaire du numéro

Éditeur(s)

Réseau Villes Régions Monde

ISSN

1916-4645 (numérique)

Découvrir la revue

Citer cet article

Lord, S., Cassiers, T. \& Gerber, P. (2014). L'impact des migrations internationales et des mobilités résidentielles sur l'évolution socio-spatiale des agglomérations de Luxembourg et Bruxelles. Environnement urbain / Urban Environment, 8, c1-c22. https://doi.org/10.7202/1027743ar
Résumé de l'article

Les inégalités sociales et spatiales n'ont cessé de croître depuis les dernières décennies dans plusieurs aires métropolitaines en Europe, comme Bruxelles et Luxembourg. L'immigration sélective entrante et sortante peut, d'une part, jouer un rôle de levier par lequel la ségrégation se renforce et, d'autre part, être un processus initiant la gentrification et la dispersion spatiale. Nous analyserons ici les dynamiques que la mobilité résidentielle et l'immigration internationale entrainent en matière de croissance des inégalités, notamment en observant la diffusion de différents groupes sociaux sur ces territoires. Le contexte économique postfordiste de compétition sera au centre de notre questionnement, et plus particulièrement son rôle comme moteur de la composition sociale appliqué aux structures spatiales de Bruxelles et Luxembourg 


\title{
L'IMPACT DES MIGRATIONS INTERNATIONALES ET DES MOBILITÉS RÉSIDENTIELLES SUR L'ÉVOLUTION SOCIO- SPATIALE DES AGGLOMÉRATIONS DE LUXEMBOURG ET BRUXELLES
}

\author{
Sébastien LORD \\ Tim CASSIERS \\ Philippe GERBER
}

\begin{abstract}
I RÉSUMÉ
Les inégalités sociales et spatiales n'ont cessé de croître depuis les dernières décennies dans plusieurs aires métropolitaines en Europe, comme Bruxelles et Luxembourg. L'immigration sélective entrante et sortante peut, d'une part, jouer un rôle de levier par lequel la ségrégation se renforce et, d'autre part, être un processus initiant la gentrification et la dispersion spatiale. Nous analyserons ici les dynamiques que la mobilité résidentielle et l'immigration internationale entrainent en matière de croissance des inégalités, notamment en observant la diffusion de différents groupes sociaux sur ces territoires. Le contexte économique postfordiste de compétition sera au centre de notre questionnement, et plus particulièrement son rôle comme moteur de la composition sociale appliqué aux structures spatiales de Bruxelles et Luxembourg.
\end{abstract}

MOTS-CLÉS - Inégalités sociales, inégalités spatiales, mobilité résidentielle, immigration sélective, double immigration

\begin{abstract}
I ABSTRACT
In most European metropolitan areas of Europe - like Brussels or Luxembourg - social and spatial inequalities have been rising since the last decades. Mechanisms of selective inward and outward migration have been playing a major role of both reinforcing existing segregation structures and initiating gentrification and spatial dispersion. We analyse the dynamics caused by both residential mobility and international immigration and look at their effects on the rise of disparities, by studying the diffusion of different social groups in the metropolitan areas. Against the background of post-fordist competitive mechanisms we will thus question the role of residential movements as a motor of social composition and spatial structure in Brussels and Luxembourg.
\end{abstract}

KEYWORDS - Spatial inequalities, social disparities, residential mobility, selective immigration, dual immigration

- Coordonnées des auteurs : Sébastien LORD, Institut d'urbanisme, Faculté de l'aménagement, Université de Montréal, Québec, Canada, sebastien.lord@umontreal.ca;Tim CASSIERS, Division of Geography, Katholieke Universiteit Leuven, CEPS/INTEAD, Centre de recherche public, Luxembourg; Philippe GERBER, CEPS/INSTEAD, Centre de Recherche Public, Luxembourg, CNRS UMR 7362 Université de Strasbourg.

ENVIRONNEMENT URBAIN / URBAN ENVIRONMENT, volume I, 20I4, p. c-I à c-22 


\section{INTRODUCTION}

La ville concentre une part croissante de la population et de la consommation énergétique. Les villes maghrébines se caractérisent par un fort essor démographique et migratoire. En Tunisie, la population urbaine est passée de $37,5 \%$ à $66,9 \%$ ' entre 1960 et 2009. Ce mouvement d'urbanisation, corrélé à la croissance économique nationale, induit des besoins accrus en matière énergétique et des impacts environnementaux démultipliés (Ministère de l'Environnement et de l'Aménagement du Territoire, 1995).

Les inégalités n'ont cessé de croître depuis les dernières décennies dans plusieurs pays européens (Atkinson et Marlier, 20l0). En outre, on observe dans de nombreuses aires métropolitaines que ces inégalités se manifestent aussi bien socialement que spatialement, en prenant diverses configurations selon les dynamiques locales, régionales et internationales en jeu. À Bruxelles par exemple, les inégalités ont évolué dans l'espace selon des processus historiques dans lesquels les mécanismes du marché du logement ont joué un rôle fondamental (Kesteloot, 1999). Au Luxembourg, et plus particulièrement dans la capitale et son agglomération, on observe des processus relativement similaires; par contre, peu de dynamiques ségrégatives peuvent être associées à l'exclusion ou à la marginalisation sociale. En revanche, on constate que la répartition dans l'espace des différents groupes sociaux semble se structurer par le haut de l'échelle sociale, où la capitale et sa première couronne périurbaine sont investies par les groupes les mieux positionnés, que d'aucuns nomment encore immigration dorée (Fehlen, 2009).

Si ces inégalités font d'emblée référence aux enjeux complexes et multiples que pose la ségrégation urbaine, touchant généralement des populations précaires, ces mêmes inégalités concernent également des processus dictés par la distinction territoriale de groupes sociaux bien positionnés socioéconomiquement. Ces enjeux fondamentaux dans l'analyse des dynamiques métropolitaines peuvent alors être abordés selon le positionnement et la mobilité des individus et des ménages dans l'environnement socio-spatial, où l'accès, la stabilité et les mouvements dans le système résidentiel (Dureau, 2002) des individus ne sont pas également partagés en matière de possibilités d'intégration et de participation. Cet article vise alors à explorer ces

'The World Bank [En ligne],Urban Population, URL : http://data.worldbank.org/indicator/SP.URB.TOTL.IN.ZS? order=wba pi_data_value_2008\%20wbapi_data_value $\% 20$ wbapi_data_valuefirst\&sort=asc, page consultée le 25 février 2013. inégalités sociales et spatiales à la lumière des mouvements résidentiels au regard de deux capitales européennes, soit Luxembourg et Bruxelles. Dans les dernières décennies, ces deux agglomérations ont en effet été fortement marquées par les pressions démographiques, où les croissances économiques et urbaines ont été rythmées par d'importants flux migratoires. Plus spécifiquement, nous proposons d'explorer la façon dont la mobilité résidentielle et l'immigration internationale contribuent aux structures d'inégalités au sein de ces deux capitales européennes. Les données exhaustives à l'échelle individuelle, retravaillées au sein du projet de recherche DESTINY (cf. Eggerickx et al., 2009), ont été utilisées pour analyser les mouvements résidentiels de l'ensemble de la population résidant dans les deux agglomérations en 2001 .

Dans un premier temps, nous questionnons les relations entre l'immigration et les structures d'inégalités à Luxembourg et à Bruxelles. À Luxembourg, il s'agit d'explorer comment ces inégalités se sont structurées suivant des tendances « classiques » de suburbanisation, alors qu'à Bruxelles il s'agit davantage de montrer la manière dont les inégalités suivent des structures historiques plutôt stables. Dans un deuxième temps, nous développons une analyse descriptive des territoires, où l'évolution générale des structures territoriales des inégalités est exposée. Dans un troisième temps, les mouvements et les dynamiques sociales sont exposés de manière à relever dans les deux agglomérations certains groupes sociaux et territoires marqués par la stabilité, la mobilité ou l'immigration, à l'aide entre autres de modèles statistiques explicatifs qui mettent en relief l'importance plus ou moins forte des mouvements de population dans les inégalités socio-spatiales. Ces modèles nous permettront alors de dégager certains résultats impliquant ou non des effets socio-spatiaux au niveau des différentes entités spatiales prises en considération dans les deux pays européens.

De manière générale, pour les deux agglomérations, il est permis d'avancer que les groupes sociaux les mieux positionnés n'ont pas de problèmes de stabilité résidentielle, ni d'accès aux territoires qu'ils voudraient choisir pour un déménagement. Pendant que les groupes moins bien positionnés peuvent rencontrer davantage de difficultés, différentes trajectoires résidentielles devraient être observées. Ces dernières trajectoires mèneraient à une dispersion inégale des groupes sur le territoire. Le positionnement sur le continuum social, influençant la facilité à déménager autant que la stabilité, devrait se refléter dans d'éventuelles dynamiques de répartition. C'est à ce titre que l'impact de l'immigration risque 
d'être le plus visible, notamment à Bruxelles. Cela dit, les immigrants qualifiés, appartenant aux groupes les mieux positionnés, risquent également de contribuer à autant de mécanismes ségrégatifs, ces derniers étant orientés vers le haut du continuum social.

\section{I. ÉVOLUTION COMPARÉE DES CONDITIONS SOCIOÉCONOMIQUES DANS LES AGGLOMÉRATIONS DE LUXEMBOURG ET DE BRUXELLES}

L'immigration est au centre de la croissance économique et du développement social des capitales européennes luxembourgeoise et bruxelloise, et ce depuis de nombreuses décennies (Besch, 2006; Montebello, 200I). L'importance de l'internationalisation dans leur développement est fondamentale, tout particulièrement à Bruxelles (Corijn et al., 2009; Vandermotten et al., 2009). Cela dit, cette immigration ne concerne pas uniquement les élites internationales mais également une immigration ouvrière. Ces processus migratoires sont conséquemment sources de problèmes d'insertion économique (Corijn et al., 2009; pour le Luxembourg, Fehlen, 2009). Dans le cas de Luxembourg-ville, en plus de l'apport quotidien d'un imposant nombre de travailleurs frontaliers (Schmitz et Gerber, 20II), l'arrivée soutenue de travailleurs étrangers assure une certaine stabilité sociodémographique et économique (Langers, 2005). À Bruxelles, la présence des institutions européennes a propulsé la ville, dans les années 1980 et 1990, à l'échelle des villes mondiales, lui conférant un statut largement supérieur à ce qu'on lui attribuerait sur la simple base de sa taille démographique. L'immigration internationale, liée directement à cette présence européenne mais également en relation avec le positionnement de Bruxelles dans les réseaux internationaux, a fait basculer les évolutions sociodémographiques que l'agglomération avait connues depuis les années 1960 (Deboosere et al., 2009). La gestion de la croissance démographique y est devenue un défi social majeur.

\section{I.I Migration et mobilité en question à l'échelle des agglomérations}

Ces flux migratoires suscitent plusieurs questions liées à l'intégration d'un nombre important d'étrangers résidant dans ces agglomérations, comptant pour près de la moitié de la population résidente (Fleury, 2009) dans le cas de Luxembourg et de $28,1 \%(55,2 \%$ si on compte la nationalité d'origine) dans le cas de Bruxelles (Willaert, 2010). Face à cela, bien que dans cet article nous n'entrions pas dans le vaste et complexe débat sur « l'intégration » des immigrants à leur société d'accueil, nous proposons néanmoins une exploration d'une assise indispensable à ce processus : ce que Dureau (2002) désigne comme le système résidentiel.

Dans ce contexte, l'immigration internationale entre dans un système d'équilibres dynamiques qui met en jeu des contrastes entre le positionnement socioéconomique des populations nationales et étrangères, mais également leur répartition sur le territoire métropolitain. À l'instar d'autres milieux métropolitains, on retrouve à Luxembourg et Bruxelles d'importants écarts entre certains sousgroupes d'étrangers - migration dorée (Fehlen, 2009) hautement qualifiée opposée à celle moins formée et ouvrière (Gerber et Pigeron-Piroth, 2009; Weiss, 2005; Corijn et al., 2009; Vandermotten et al., 2009). Ces écarts s'ajoutent aux écarts socioéconomiques déjà présents dans la structure sociale de la population nationale. Certaines classes sociales, étrangères ou non, se trouvent ainsi défavorisées face à d'autres en termes de capitaux économiques, sociaux et culturels (Bourdieu, 1979). C'est du moins ce qui a déjà été observé à l'échelle nationale pour le Luxembourg et pour la Belgique en matière de positionnement des ménages dans le système résidentiel ainsi que sur le territoire (Lord et Gerber, 2012; Lord et Gerber, 2009; Kesteloot et al., 2006).

L'exploration originale de l'immigration proposée ici prend appui sur la dimension longitudinale et prend le territoire des agglomérations (centre, banlieue et périphérie) de Luxembourg et de Bruxelles comme unité d'analyse. Non seulement l'immigration internationale est fondamentale dans la structure sociodémographique de ces deux agglomérations, mais ces capitales européennes constituent un lieu de passage stratégique pour les flux migratoires à l'échelle des deux pays. C'est une exploration systématique qui est fondée sur le dernier recensement national exhaustif de 200I des deux pays disponibles, de manière à suivre l'évolution des deux agglomérations du point de vue socioéconomique. Sous une question centrale qui s'intéresse aux mouvements résidentiels des populations nationales et étrangères plus ou moins bien positionnées, deux objectifs sont ici visés: I) comprendre l'interaction entre les mobilités résidentielles et les structures d'inégalités; 2 ) isoler le rôle de l'immigration internationale dans la structuration des inégalités présentes dans les deux agglomérations.

Plus précisément, deux types distincts de mobilité au sein du système résidentiel sont ici explorés, rejoignant la distinction proposée par Kaufmann (2005) entre, d'un côté, un déplacement dans l'espace 
et, de l'autre, un déplacement au sein d'une certaine échelle sociale. L'auteur s'appuie sur les concepts de «mobilité spatiale», de «fluidité sociale», une conception reprise par Bauman (2000) et basée sur le concept de «modernité liquide ». La typologie de Kaufmann (2000) permet d'apporter une distinction pertinente en séparant différentes mobilités géographiques selon des critères spatio-temporels (tableau I).

L'échelle temporelle oppose d'un côté les déplacements cycliques qui supposent, explicitement, une répétition régulière et, implicitement, un retour au point de départ, et, de l'autre, les déplacements linéaires, qui eux ne nécessitent pas de retour. La première temporalité renvoie ainsi au quotidien et la seconde au cycle de vie, à l'origine de nombreuses mobilités résidentielles. II est ici question d'explorer et de distinguer les mouvements linéaires, à savoir la mobilité résidentielle et la migration, au sein d'inégalités structurées selon un continuum social.

\section{I.2 Les processus de métropolisation sur Luxembourg et Bruxelles}

Au Luxembourg, avec ce que plusieurs auteurs observent comme le début de la métropolisation de la capitale luxembourgeoise (Sohn et al., 2009), on note une croissance économique et démographique remarquablement soutenue du Grand-Duché. Sans entrer dans la problématique transfrontalière, incontournable lorsqu'il est question de l'agglomération luxembourgeoise, l'aire d'attraction métropolitaine de Luxembourg-Ville ne peut être passée sous silence en s'étendant bien au-delà des frontières du pays, notamment visible avec la présence de plus de 130.000 travailleurs-navetteurs transfrontaliers (Schmitz et Gerber, 20II). La périurbanisation du territoire de l'agglomération de Luxembourg représente de manière explicite la matérialisation graduelle du processus de métropolisation, mettant d'autant des pressions foncières et immobilières à l'intérieur, voire à l'extérieur, du territoire luxembourgeois (Gerber et al., 20/2). Malgré cette croissance généralisée de l'économie, qui s'est entre autres manifestée par le phénomène de gentrification de quelques quartiers centraux de Luxembourg-Ville (Gerber et Pruvot, 2005), nous avançons l'hypothèse que cette croissance n'a pas été uniforme au sein de l'agglomération de la capitale luxembourgeoise. En outre, elle s'est effectuée au profit de certains groupes sociaux composant les communautés résidentes suburbaines.

À Bruxelles, malgré des évolutions économiques favorables, le taux de chômage et les inégalités sociales n'ont cessé de croître (Van Hamme et al., 20l I). Depuis les années 1990, on observe dans cette capitale un renouveau économique tertiarisé caractéristique des processus de métropolisation décrit un peu partout dans le monde (Van Hamme et al., 20II). Pourtant, bien que le centre de l'agglomération performe mieux que la moyenne du territoire belge, une grande partie de la croissance économique de l'aire métropolitaine bruxelloise est concentrée dans les espaces périurbains. Ainsi, contrairement à Luxembourg, les bonnes performances économiques du centre de l'agglomération bruxelloise ne seraient pas à l'origine d'une périurbanisation résidentielle, mais s'accorderaient avec une longue histoire d'étalement urbain, qui touche aussi bien les entreprises (D'Andrimont et al., 2007) que la population (Deboosere et al., 2009).

Donzelot et Jaillet (1997) proposent un modèle afin de comprendre la relation entre ces mouvements linéaires et le positionnement social à travers la structuration socio-spatiale des aires métropolitaines. Cette structuration est ainsi le résultat d'un contexte structurel historique. Pendant le régime fordistekeynesien, les villes fonctionnaient comme les moteurs des économies nationales. Ces villes constituaient un continuum socio-spatial liant les différentes zones résidentielles; le progrès social était incorporé au système productif et se traduisait dans des trajectoires

Tableau I - Les différentes mobilités géographiques

\begin{tabular}{|l|cc|}
\hline & $\begin{array}{c}\text { Mouvement interne à un } \\
\text { bassin de vie }\end{array}$ & $\begin{array}{c}\text { Mouvement vers l'extérieur } \\
\text { d'un bassin de vie }\end{array}$ \\
\hline Mouvement cyclique & Mobilité quotidienne & Voyage \\
Mouvement linéaire & Mobilité résidentielle & Migration \\
\hline
\end{tabular}

Source : Kaufmann, 2000 
spatiales de la campagne vers les quartiers industriels et les zones suburbaines. Avec l'avènement de la mondialisation, les villes sont devenues des cités globales, échappant au cadre national. Ces villes ont en quelque sorte rompu avec la logique du continuum socio-spatial. Le centre et la périphérie ne font plus partie d'un seul système; ils impliquent dorénavant des fragmentations socio-spatiales. Le centre se met en réseau avec des autres centralités urbaines à l'échelle mondiale, pendant que la périphérie se développe de manière autonome. La mobilité résidentielle du centre vers la périphérie peut alors être comprise comme une fuite de la ville, pendant que le trajet inverse peut être motivé par des intentions d'intégrer l'élite cosmopolitaine mondiale.

La question centrale ici est alors de savoir si l'on peut observer, pour le cas des espaces métropolitains luxembourgeois et bruxellois, ce changement d'organisation fordiste-keynesienne vers un modèle répondant aux logiques de flexibilité liées à la mondialisation. Afin d'explorer cette question, nous examinons d'abord l'évolution des structures spatiales dans les deux capitales, pour ensuite analyser la façon dont les mobilités résidentielles et l'immigration des différents groupes sociaux se rapportent à l'un ou l'autre de ces modèles.

\section{L'ÉVOLUTION DES INÉGALITÉS DANS LES CONDITIONS SOCIOÉCONOMIQUES}

Une recherche nous a amenés à explorer l'évolution spatiale et temporelle des inégalités au Luxembourg et en Belgique (projet DESTINY, cf. Eggerickx et al., 2009; Lord et Gerber, 2013) de manière comparative. Les données des derniers recensements exhaustifs de 199I et de 200I des deux pays, ainsi que celles des enquêtes-panels européens de 1995 et de 2003 ont été utilisées pour construire deux typologies socioéconomiques des ménages et des territoires. Ces dernières témoignent, pour l'ensemble du Luxembourg et de la Belgique, de l'amélioration des conditions socioéconomiques de la population entre ces deux recensements. Nous prenons donc appui sur ces travaux pour développer cette comparaison du rôle des migrations internationales sur l'évolution des structures d'inégalités dans ces deux capitales européennes.

\section{I Une perspective sociale des inégalités au Luxembourg et en Belgique}

D'un point de vue méthodologique, des données de panel ont donc été utilisées pour mesurer le niveau de vie (revenus monétaires) au niveau individuel, ainsi que pour le ménage, afin de quantifier un continuum social. Au Luxembourg, deux vagues différentes du PSELL (Panel Socio-Économique Liewen zu Lëtzebuerg) ont été couplées aux données des recensements, 1995 pour le recensement de 199| et 2003 pour celui de $2001 .^{2}$ Les questionnaires de ces enquêtes comportent des parties individuelles et d'autres concernent l'ensemble du ménage, parmi lesquelles le revenu total du ménage, quel que soit sa provenance (salariale, allocation de chômage, de retraite, etc.). Pour positionner chacun des individus dans les recensements exhaustifs, nous avons utilisé la formule désormais éprouvée du RDUC (revenu disponible selon les unités de consommation), qui est le rapport entre le revenu total du ménage et le nombre pondéré d'individus le constituant. Ce revenu moyen a été calculé pour chacune des modalités communes aux deux bases de données, puis mathématiquement standardisé. On obtient alors un score (positif ou négatif, où la moyenne standardisée est donc de 0 et l'écart-type de I) pour chacune des modalités des trois dimensions sociales, score qui sera différent pour les individus en 1991 et en 200I.

En fonction des réponses (modalités) aux deux recensements pour ces trois dimensions ${ }^{3}$ (éducation ${ }^{4}$, catégories socioprofessionnelles ${ }^{5}$ et logement $t^{6}$ ), le score standardisé correspondant à chacune des modalités des trois dimensions a été réaffecté au sein de la base de données exhaustive. À titre d'exemple, la figure I présente la distribution des scores en fonction des modalités de la dimension " niveau d'éducation ». On y voit que la plupart des modalités sont caractérisées par un score négatif, c'est-à-dire que les individus ayant donné ces réponses lors de l'enquête EU-SILC avaient un RDUC inférieur à la moyenne 0 .

\footnotetext{
${ }^{2}$ Pour une description détaillée de la méthode développée pour cette étape du projet DESTINY, voir : Lord et al. (20II).

${ }^{3}$ Le choix de ces dimensions s'est effectué en lien avec l'approche du mode d'intégration économique de Karl Polanyi, dont les concepts sont utiles pour comprendre la pauvreté et les inégalités, mais aussi pour caractériser les changements économiques et politiques.

${ }^{4}$ Les modalités pour le niveau d'éducation sont les suivantes : sans diplôme, primaire, technique et professionnel, secondaire général, secondaire supérieur / universitaire.

${ }^{5}$ Chômeur, ouvrier, à la maison, retraité, inactif, autre inactif, indépendant / professionnel, employé du public, employé du privé.

${ }^{6}$ Pour le logement, les modalités sont cumulatives : insalubre (locataire ou propriétaire), salubre sans confort (locataire ou propriétaire); téléphone et au moins une pièce par personne (locataire ou propriétaire), avec chauffage central (locataire ou propriétaire), avec au moins deux pièces par personne (locataire ou propriétaire), avec ordinateur (locataire ou propriétaire). Les individus « logés gratuitement » ont été regroupés avec les locataires.
} 
Seuls les diplômés de l'enseignement supérieur avaient un revenu (largement) plus élevé que cette valeur pivot (0). A l'opposé, ce sont logiquement les individus les moins bien scolarisés (primaire, technique et professionnel) ou non diplômés pour lesquels on observe les scores les plus négatifs / défavorables, car leurs RDUC sont très en-dessous de la moyenne. Les individus du secondaire, pour leur part, se situent aux environs de la moyenne.

Sur la base de ces trois scores individuels, la formation des groupes sociaux a pu être effectuée pour les deux pays. Dans un premier temps, l'ensemble de la population a été regroupé à l'aide d'une classification hiérarchique ascendante réalisée avec l'ensemble des combinaisons des modalités des trois dimensions (pour rappel, éducation, catégorie socioprofessionnelle et logement). Les individus partageant des conditions socioéconomiques similaires ont ainsi été agrégés en 17 groupes pour le Luxembourg $(n=280432)$ et 16 pour la Belgique $(n=7440$ II5) en 200I. Les populations résidant dans les deux pays ont été classées et ordonnées en groupes sociaux, allant des moins bien positionnés aux plus favorisés (figure 2).

Figure 1 - Exemples des scores de la dimension éducation, calculés avec l'EU-SILC et transposés dans les recensements belges et luxembourgeois
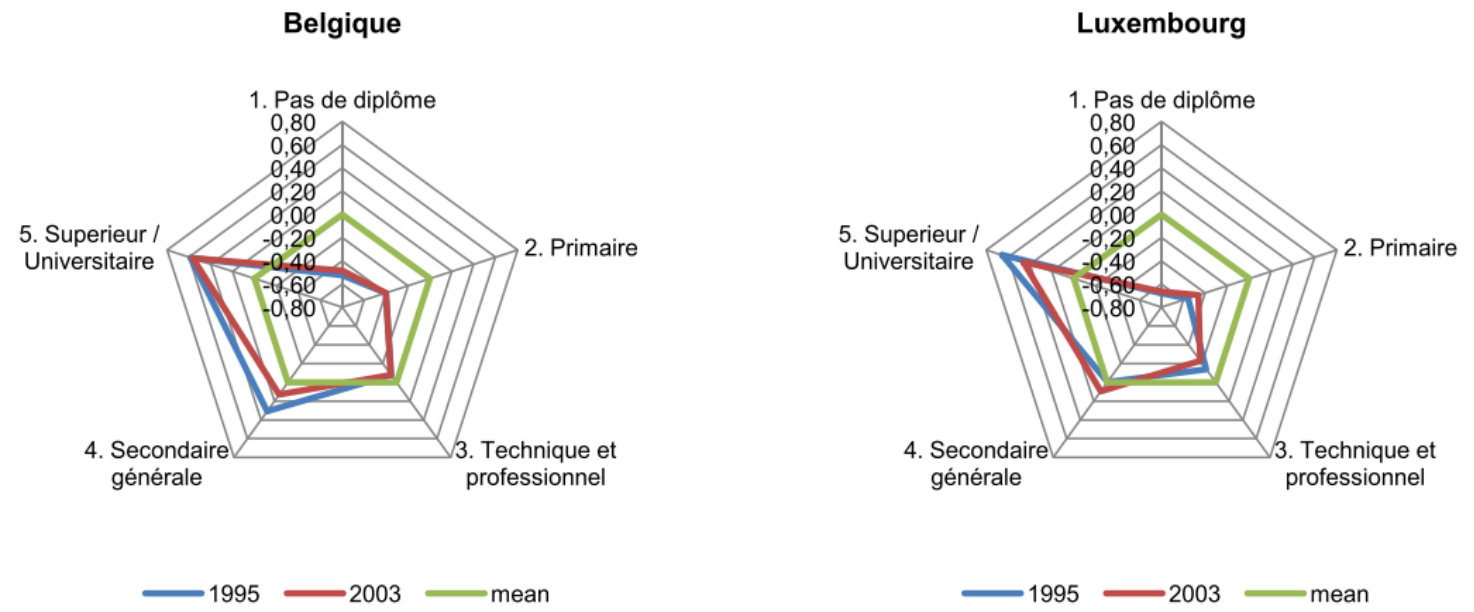

Sources: Statec, recensement général de la population, 2001; INS, Enquête socio-économique générale, 2001; EU-SILC, 1995,2003

Figure 2 - Distribution des groupes sociaux du plus précarisé au plus favorisé au Luxembourg et en Belgique, 2001 (\%)

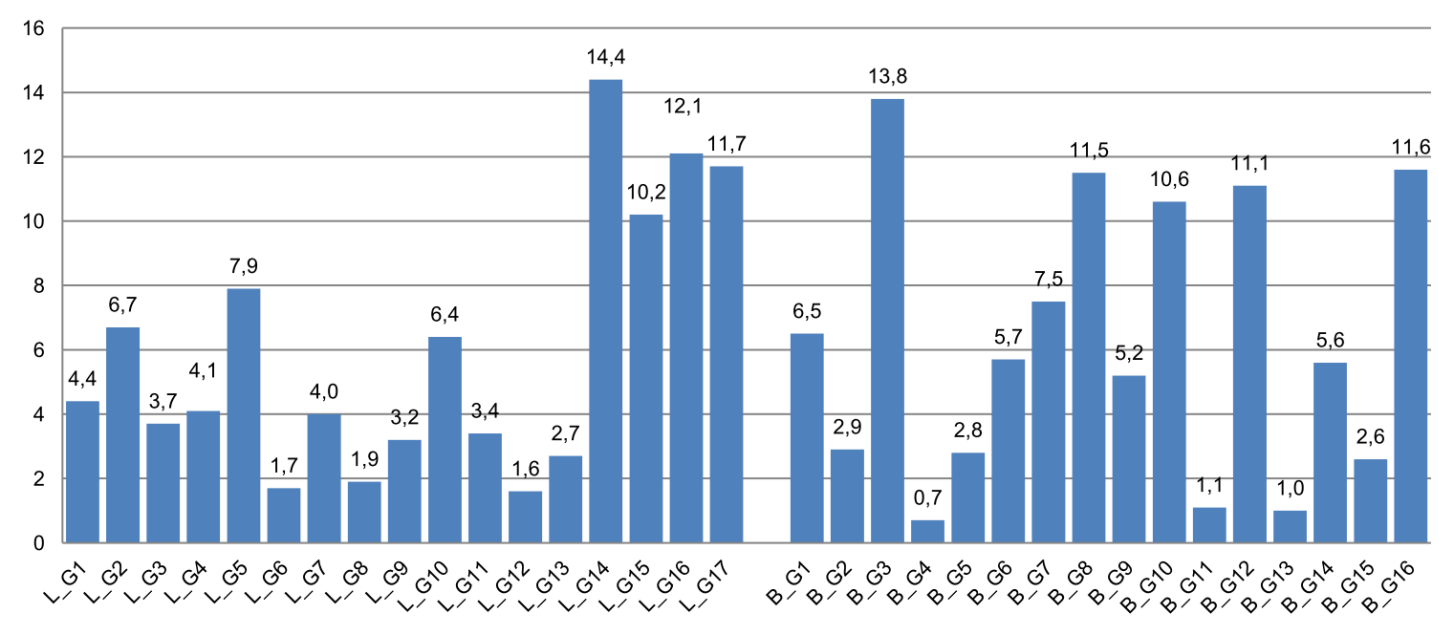




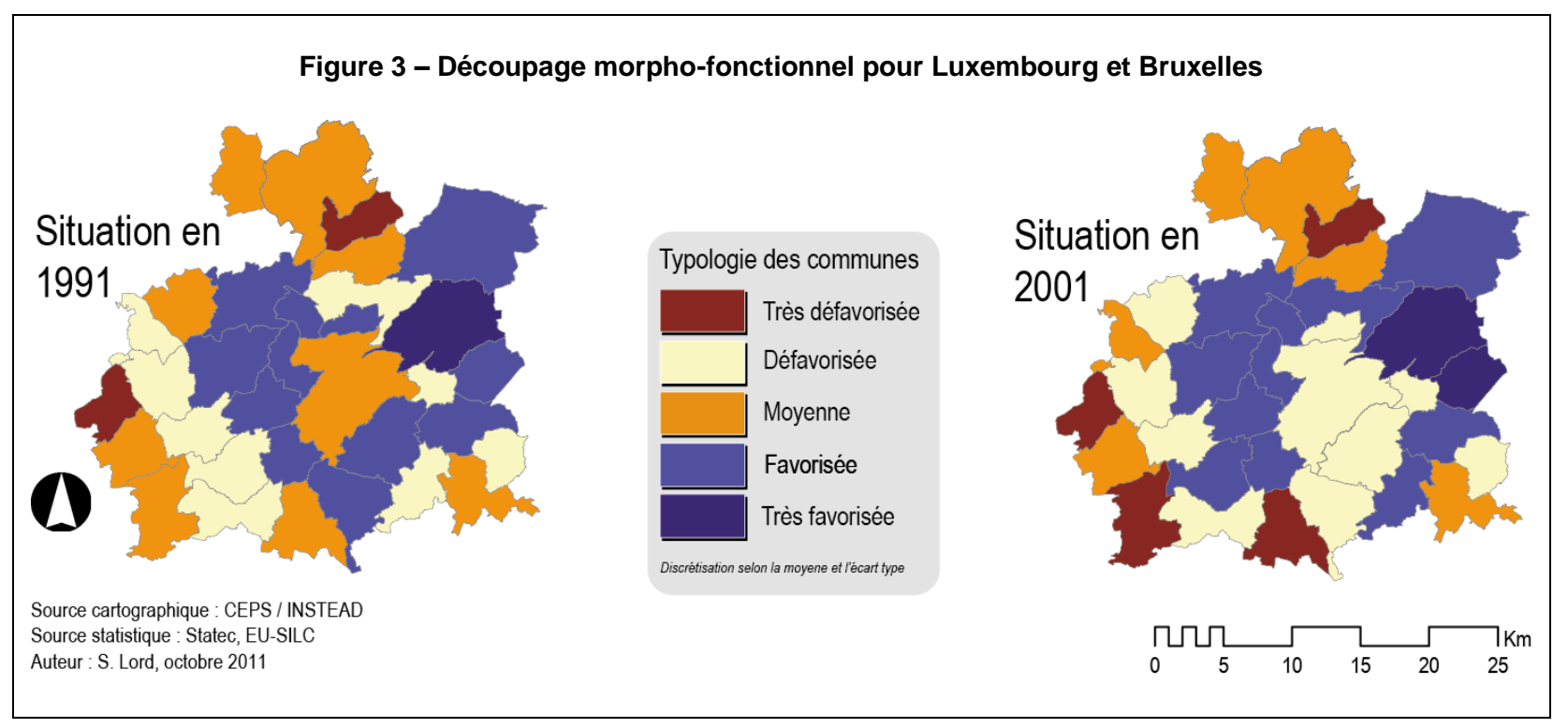

\subsection{Une approche territoriale des inégalités à Luxembourg et Bruxelles}

Pour réaliser l'analyse géographique de la répartition de ces groupes sociaux, au Luxembourg, nous avons utilisé une question présente dans le recensement luxembourgeois de $200 \mathrm{I}$ qui permet de rendre compte de la dimension longitudinale de la mobilité résidentielle au sein de l'ensemble des communes, et donc d'informer certaines tendances au niveau des acteurs des différents déplacements résidentiels sur le territoire. Cette question identifie le lieu de résidence de chacun des individus présents au recensement au $\mathrm{I}^{\text {er }}$ janvier 1995, donc six années avant le recensement de 200I. Pour la Belgique, nous avons croisé les données du recensement de $200 \mathrm{I}$ avec les données du registre national de 1996. Ces données nous ont ainsi permis d'observer les distributions de la population en 2001 et en 1995 voire 1996, et par la suite de construire des matrices de transition 1995/I996-200I pour trois types de trajectoires dans le système résidentiel : I) stabilité résidentielle, 2) la mobilité résidentielle (nationale) et 3 ) l'immigration internationale. Ce suivi longitudinal a été effectué, comme le montre la figure 3 , sur la base d'un découpage morpho-fonctionnel ${ }^{7}$ structurant le territoire des deux agglomérations entre trois types d'espaces : I) centre, 2) banlieue, 3) périphérie.

\footnotetext{
${ }^{7}$ Pour le Luxembourg, la typologie proposée par Carpentier (2006) a été utilisée. Elle est issue de croisements factoriels sur les caractéristiques du bâti, le zonage fonctionnel ainsi que sur des flux domicile-travail vers la capitale. Pour Bruxelles, nous avons utilisé la typologie de Luyten et Van Hecke (2007) qui se base sur le même type de variables.
}

\section{EXPLORATION DES DYNAMIQUES SOCIALES ET TERRITORIALES}

Les prochaines sections proposent une analyse descriptive des territoires, où l'évolution générale des structures territoriales des inégalités est exposée. Cette nécessaire description est suivie d'une analyse des mouvements et des dynamiques sociales de manière à relever dans les deux agglomérations certains groupes sociaux marqués par la stabilité, la mobilité résidentielle ou encore l'immigration.

\section{I Structures des inégalités à Luxembourg et Bruxelles en 1991 et en 200 I}

Dans un premier temps, afin de présenter globalement l'évolution des structures d'inégalités des agglomérations luxembourgeoise et bruxelloise, nous avons construit deux typologies territoriales. L'ensemble de la population a été considérée selon la commune de résidence en $199 \mathrm{I}$ et en $200 \mathrm{I}$ pour le Luxembourg et selon le quartier de résidence en 1991 et $200 \mathrm{I}$ pour Bruxelles, où une moyenne a été calculée pour chacune des trois dimensions que sont l'éducation, la catégorie socioprofessionnelle et le logement. Dans un deuxième temps, une analyse en composantes principales a permis de dégager un score multidimensionnel pour chacune des unités spatiales des deux agglomérations. Cette composante a été cartographiée pour les deux situations (I99| et 200I) selon une discrétisation classique par la moyenne et l'écart-type. 

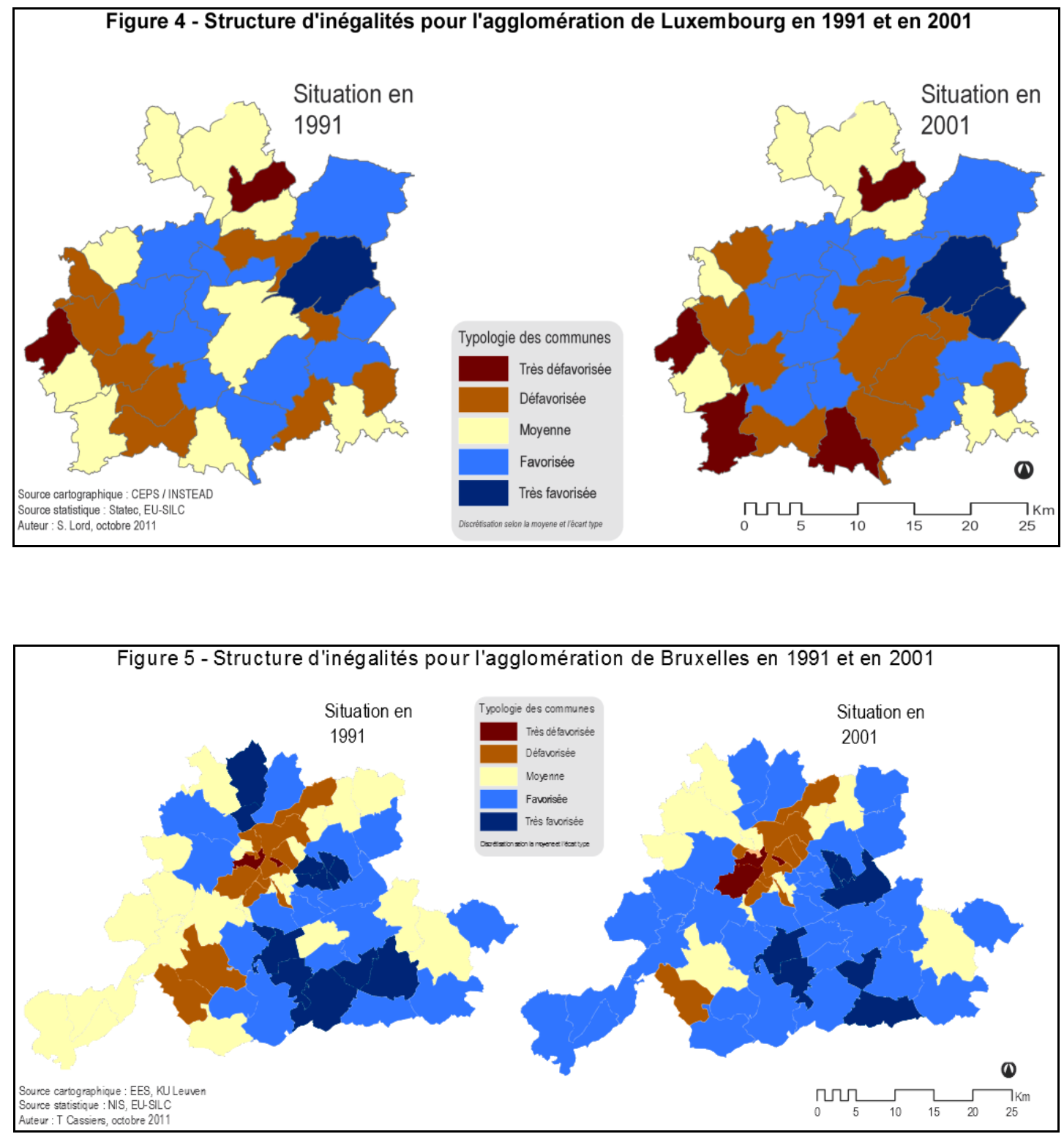

La figure 4 montre que, globalement au niveau de l'agglomération de Luxembourg, certaines similitudes apparaissent entre les situations de 199I et de 200I. Une forte polarisation du haut de l'échelle sociale est visible autour du centre de la capitale, et plus spécifiquement dans sa périphérie périurbaine rapprochée. La comparaison des deux typologies indique que cette polarisation semble s'être renforcée dans l'intervalle 199I-200I. En 200I, on observe davantage de communes très défavorisées et très favorisées en deuxième couronne de banlieue, alors que le centre est passé dans la catégorie défavorisée. Cette structure, où le basculement des communes du sud et du sud-ouest de l'agglomération vers les catégories défavorisées, semble s'opérer en lien avec l'évolution sociodémographique observée dans l'ancien bassin minier du sud du pays où la précarité semble davantage se concentrer (Lord et Gerber, 2009; Gerber et Piroth, 2004). 
Pour Bruxelles (figure 5), les structures n'ont pas fortement changé entre 199I et 200I. Une forte polarisation est visible entre les communes centrales très défavorisées dans la Région Bruxelles-Capitale et les communes dans la périphérie, le reste de la Région étant plutôt dans une position moyenne. Malgré une diminution du nombre de communes très favorisées, la structure d'inégalités est encore plus prononcée en 200I. Tandis qu'en 199/ la périphérie ressemble plutôt à une mosaïque positionnée autour de la moyenne, en $200 \mathrm{I}$ elle se présente comme plus homogène et favorisée. Les communes centrales restent (très) défavorisées, même si on constate une tendance d'élargissement de cette zone vers l'est dans la Région de Bruxelles-Capitale, tout comme vers le nord et le sud en suivant l'ancien axe industriel du canal. Cela suggère que la gentrification pourrait s'attaquer aux communes centrales en provenance des communes plus favorisées dans l'ouest (Van Crieckingen, 2009).

D'un point de vue social, ces structures d'inégalités des agglomérations luxembourgeoise et bruxelloise se reflètent aussi dans les distributions des groupes sociaux construits pour l'ensemble des deux pays. Comme le suggère la figure 6 , l'agglomération luxembourgeoise affiche une faible représentation des groupes les plus précarisés (moins de $5 \%$ ) (groupes I à 3 ), ces derniers étant davantage présents dans le reste du pays, notamment au sud. Ce sont les groupes sociaux moyens et supérieurs (plus de 55\%) (groupes 10 à 15) qui y sont finalement les plus présents. Le cas luxembourgeois se distingue également par une forte polarisation du bas et du haut de l'échelle sociale au centre, où les groupes les mieux (groupes 16 et 17 ) et les moins bien positionnés (groupe I à 3 ) partagent le même espace communal. Caractéristique de la double migration abordée plus haut, cette configuration s'applique également pour les individus n'appartenant pas à un groupe défini (missings) qui compte pour $16 \%$ de l'agglomération. Plus typiquement, les couronnes de banlieues sont celles des classes moyennes et supérieures où l'on observe également une forte présence des groupes les plus favorisés.

De manière globale à Bruxelles, les groupes en bas de l'échelle représentent $20 \%$ de la population résidente en $200 \mathrm{I}$ (figure 7). Au niveau de la répartition spatiale, Bruxelles montre une forte dualité, avec les groupes les plus précarisés (groupes I à 3 ) qui se situent dans le centre et qui sont très peu présents dans les couronnes. À l'opposé, en haut du

Figure 6 - Répartition des groupes sociaux dans l'agglomération de Luxembourg en 2001

Distribution dans l'agglomération

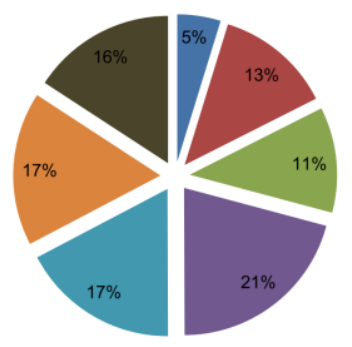

Répartition dans les différents zones (agglomération $=1$ )

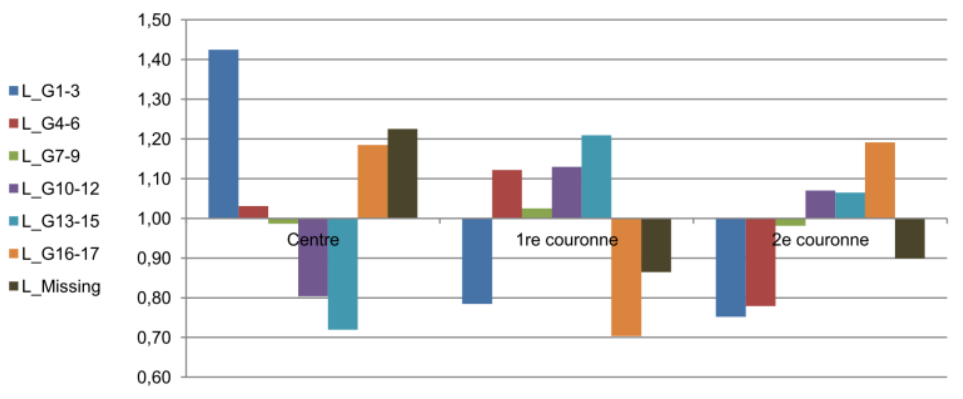

Figure 7 - Répartition des groupes sociaux dans l'agglomération de Bruxelles en 2001

Distribution dans l'agglomération

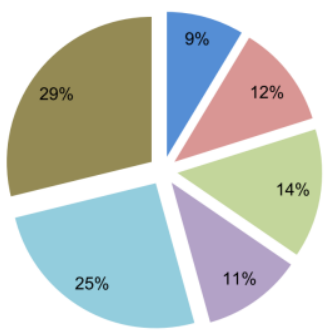

Répartition dans les différents zones (agglomération $=1$ )

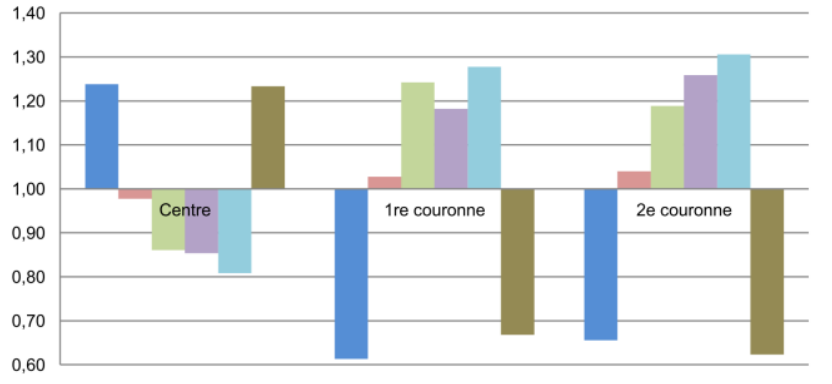


continuum social, les groupes 13 à 16 résident surtout dans les couronnes de banlieue. Les autres groupes moyens et supérieurs suivent cette structure, avec une surreprésentation claire en première et deuxième couronne. Enfin, le groupe des « missings » forme une partie importante de la population (29\%) de l'agglomération bruxelloise. Dans l'agglomération de la capitale belge, ce groupe correspond aux groupes les plus précarisés (groupes I à 3).

Ainsi, d'un point de vue social, la configuration spatiale bruxelloise est relativement différente de celle de l'agglomération de Luxembourg. Pendant que l'on observe une forte représentation des groupes moyens et supérieurs dans les couronnes de banlieues des deux capitales, caractéristiques des processus de métropolisation et de périurbanisation, au centre de Luxembourg cohabitent le haut et le bas de l'échelle sociale, alors que le centre de Bruxelles abrite une plus forte proportion des groupes les plus précaires et une plus faible proportion du haut de l'échelle sociale. Face à cela, nous constatons que les différents groupes sociaux occupent des territoires relativement différenciés.

À Bruxelles, cela résulte en une opposition forte entre le centre et les deux couronnes de banlieue. D'un côté, il y a une forte attractivité des zones périurbaines pour la population plus aisée. Les zones centrales sont investies par les groupes au bas de l'échelle. Cette structuration peut être comprise par les mécanismes de sélection historiques dans le marché du logement. En effet, l'exode de la population plus aisée des quartiers centraux de Bruxelles depuis les années 1960 a transformé la plupart des logements en habitats " résiduels » (Kesteloot, 1999), avec des prix abordables pour les groupes les plus précarisés. Manque d'alternatives, vu le faible taux de logements sociaux à Bruxelles, ceci a provoqué une concentration des groupes défavorisés (et plus particulièrement ceux issus de l'immigration ouvrière) dans ces secteurs. Au Luxembourg, et plus particulièrement dans l'agglomération de LuxembourgVille, on observe des processus relativement similaires, mais peu de marginalisation sociale. En revanche, on constate une ségrégation qui se structure par les élites, où la capitale et sa première couronne périurbaine sont investies par les groupes sociaux les mieux positionnés. Ainsi, certaines tendances ségrégatives observées dans d'autres villes européenes semblent se structurer différemment dans la capitale luxembourgeoise. En effet, on n'y retrouve pas d'autre grande agglomération dans le pays, très peu de logements sociaux, un fort pourcentage d'étrangers mais, en parallèle, peu de groupes stigmatisés ainsi qu'un très faible taux de chômage. Par conséquence, les dynamiques impliquant les groupes sociaux moins bien positionnés sont moins claires, notamment parce qu'elles concernent des territoires sur l'ensemble du petit pays et s'observent sur des zones très spécifiques du territoire, notamment dans l'ancien bassin minier au sud du pays, voire même dans les zones frontalières à l'extérieur du Grand-Duché (Lord et Gerber, 2012).

Qu'en est-il de cette répartition sociale et spatiale, risque-t-elle de se renforcer avec les trajectoires résidentielles des habitants de ces deux agglomérations? Nous nous sommes penchés sur les "possibilités» des différents groupes sociaux de changer de lieu de résidence. Au centre de ce questionnement, nous nous attardons à l'évolution qui a conduit aux structures sociales et spatiales des deux agglomérations en 200I. Nous questionnons plus précisément l'influence et le rôle des mobilités résidentielles et de l'immigration internationale sur l'évolution des structures d'inégalités observées. II s'agit d'explorer, à travers mouvements et stabilité, les positions des différents groupes dans l'espace social et spatial des deux agglomérations.

\subsection{Description des déplacements résidentiels des groupes sociaux}

Les prochaines sections examinent les déplacements résidentiels dans la période 1995-200 I pour Luxembourg et 1996-200I pour Bruxelles des groupes sociaux des deux agglomérations selon le découpage spatial proposé (figure 3). Vu que les différences en termes de structure sociale $y$ sont minimales, et pour des raisons de clarté, les deux couronnes de banlieues ont été prises ensemble dans cette analyse.

Le premier constat effectué concerne le profil de la population mobile. Dans les deux agglomérations, la position sur l'échelle sociale des individus mobiles est nettement supérieure à celle de la population stable. Cette observation va dans le même sens que nos hypothèses, notamment par rapport aux possibilités de pouvoir changer de lieu de résidence qui ne sont pas uniformément réparties dans la population. Comme le montre les figures 8 à 15 , de manière globale, en prenant l'ensemble des individus ayant changé de commune de résidence durant la période analysée dans les deux pays, nous constatons que les scores moyens révélant la position des individus sur le continuum social (pour rappel, calculés à partir d'une standardisation du RDUC issu des panels européens des ménages) varient selon à la fois la mobilité résidentielle et l'immigration, ainsi que les origines et les destinations dans l'agglomération. 


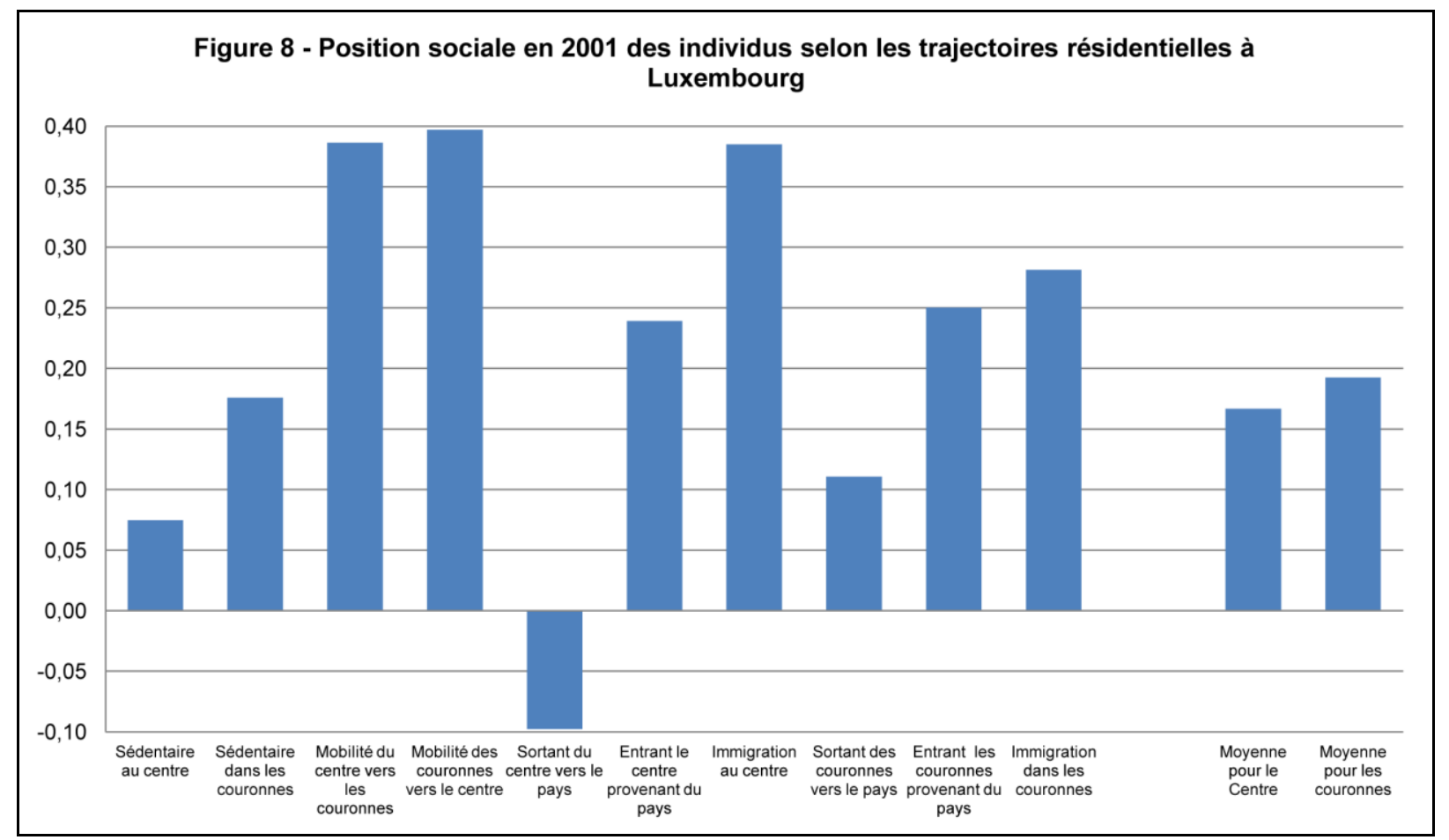

\subsection{MOBILITÉS ET IMMIGRATION À LUXEMBOURG}

Dans l'agglomération de Luxembourg (figure 8), le score moyen standardisé en 2001 de la population qui n'a pas changé de commune de résidence $(0,07$ au centre et 0,18 dans les couronnes) depuis 1995 est inférieur à celui de la population qui a déménagé, que ce soit vers les couronnes $(0,38)$ ou vers le reste du pays $(0, \mathrm{II})$. Les mobilités entrantes vers le centre, qu'elles proviennent des couronnes $(0,40)$ ou du reste du pays $(0,24)$ sont également le fait d'individus mieux positionnés.

Un même pattern est également visible pour l'immigration, où les immigrants les plus favorisés s'installent dans l'agglomération, soit au centre $(0,39)$ ou dans les couronnes $(0,28)$. Pour l'agglomération de Luxembourg, des différences significatives sont ainsi visibles au niveau des individus mobiles et des immigrants. Ces deux types de mouvements résidentiels concernent les individus les mieux positionnés sur le continuum social, se localisant également en périphérie rapprochée ou dans le centre de l'agglomération grand-ducale.
Lorsque ces trajectoires sont croisées avec les groupes sociaux (figure 9), des différences sensibles apparaissent selon le centre et les couronnes de banlieue. Environ la moitié des groupes positionnés au bas de l'échelle sociale, que ce soit au centre ou dans les couronnes, n'ont pas déménagé entre 1995-200I. Les mouvements résidentiels du centre vers les couronnes ont d'abord concerné les groupes les mieux positionnés (11\% à plus de $14 \%$ ), tandis que les mouvements vers le reste du pays surtout les groupes les plus favorisés $(9 \%)$ et les groupes moyens $(5 \%$ à $7 \%$ ), mais moins les groupes favorisés (4\%). Mise à part la plus faible stabilité des groupes défavorisés dans les couronnes, peu de différences sont observables entre les groupes pour ce type de territoire. Alors que les groupes moyens-inférieurs se distinguaient avec une plus grande stabilité au centre, une observation similaire peut se faire au niveau des groupes moyenssupérieurs dans les couronnes. Contrairement aux sorties du centre vers les couronnes, les déplacements des couronnes vers le centre sont à la fois le fait des groupes les mieux positionnés et ceux qui le sont le moins (environ 8\%). Enfin, qu'il s'agisse du centre ou des couronnes, l'immigration hautement et moins qualifiée, associée aux groupes du haut et du bas de l'échelle, se distingue aussi nettement pour le centre de l'agglomération. 

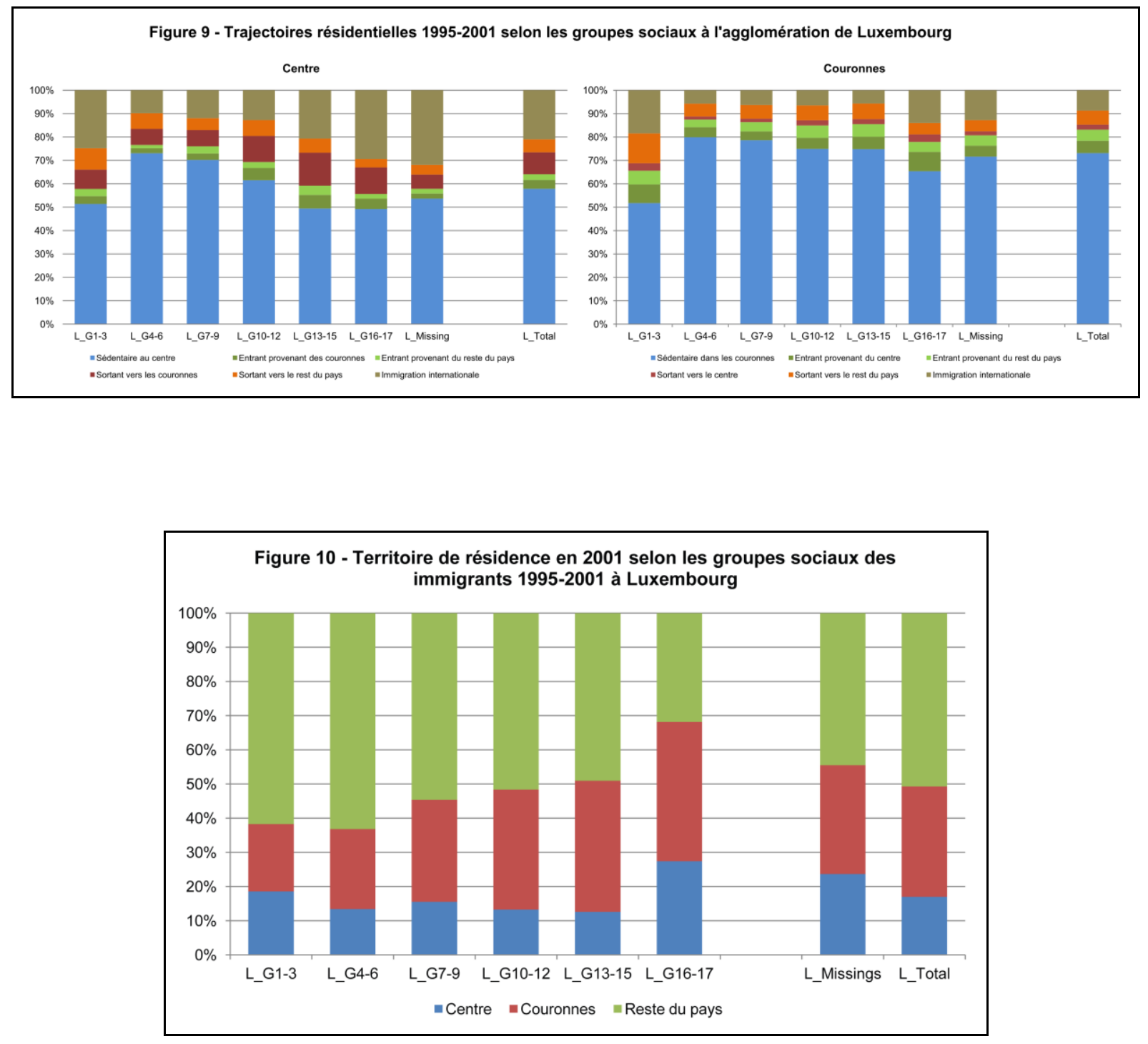

Les flux d'immigration internationale entrant dans l'agglomération luxembourgeoise semblent induire une dynamique sociale dictée par les groupes favorisés: aspirer à entrer dans l'agglomération et dans le centre concerne les mieux positionnés socioéconomiquement alors que le reste du pays concerne les groupes les moins bien positionnés. Aussi bien pour le centre que pour les couronnes, la plupart des déplacements observés dans l'agglomération grandducale, tant la mobilité résidentielle que l'immigration, est le fait des groupes les plus avantagés.

Quant à l'immigration, la figure 10 montre les territoires de résidence des immigrants en 2001 à Luxembourg selon les groupes sociaux. En 1995, le rôle de l'immigration dans les dynamiques spatiales de l'agglomération luxembourgeoise ressort nettement.
Les immigrants du haut de l'échelle sociale structurent clairement les territoires des couronnes et du centre de l'agglomération. Si les immigrants situés au bas du continuum social sont surreprésentés au niveau du centre de l'agglomération, et leur présence est relativement faible en périphérie, c'est dans le reste du pays qu'ils s'installent. En fait, la proportion des groupes sociaux présents dans les couronnes s'accroit de manière quasi linéaire suivant le positionnement de ces derniers sur le continuum social, linéarité qui ne s'observe pas dans l'agglomération bruxelloise.

\subsubsection{MOBILITÉS ET IMMIGRATION À BRUXELLES}

À Bruxelles, contrairement à Luxembourg, il a été possible d'analyser les déplacements résidentiels à l'intérieur du centre et des couronnes (figure II). En 

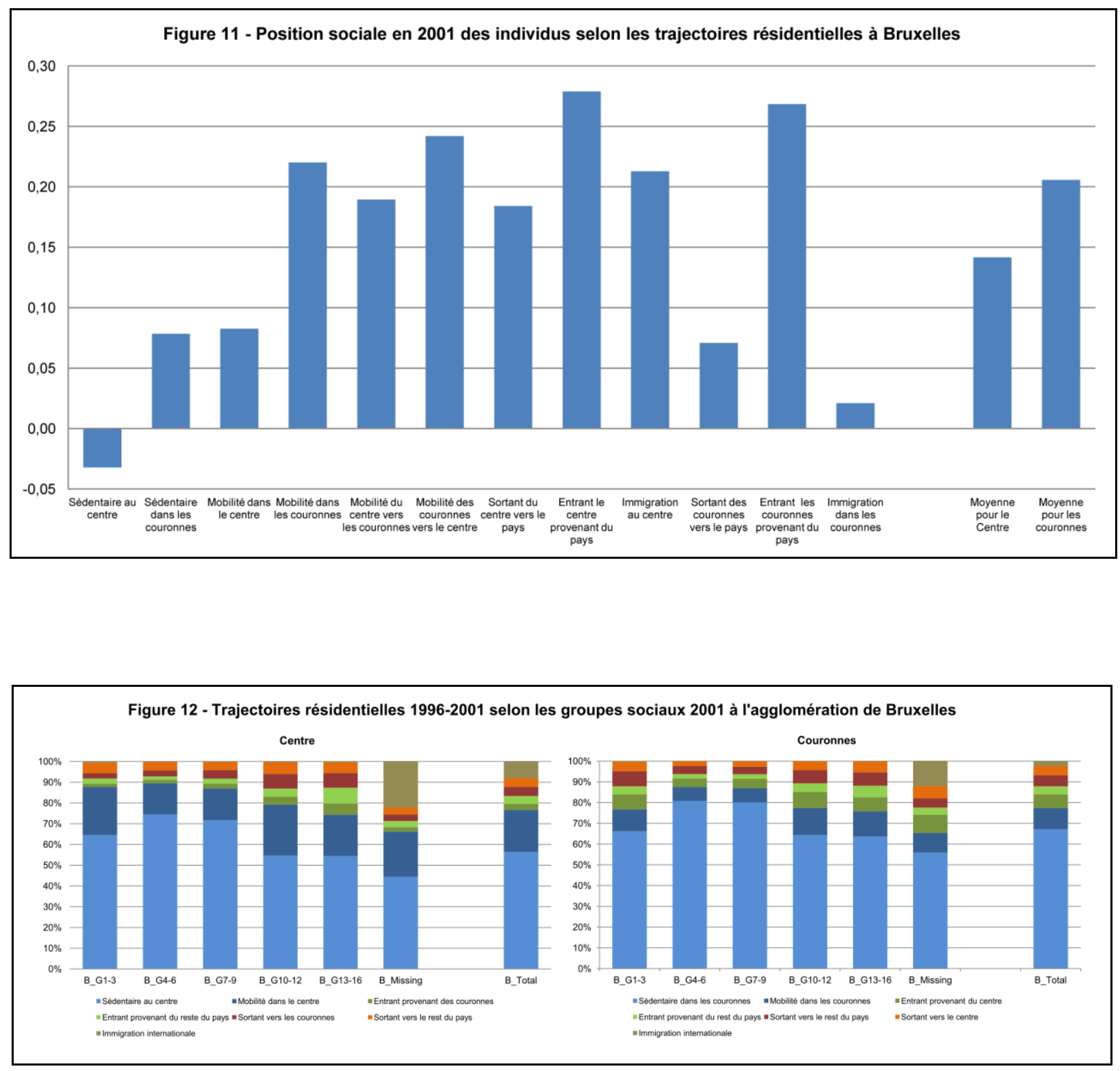

prenant en compte ces mouvements, on observe que le score moyen de la population ayant effectué un changement de résidence dans le centre $(0,08)$ est bien inférieur au score de ceux qui ont déménagé vers les couronnes ou le reste du pays (respectivement 0,19 et 0,18 ). Pour les couronnes de l'agglomération bruxelloise, il n'y a pas de différence significative entre un déplacement à l'intérieur de ces couronnes $(0,22)$ et un déplacement vers le centre $(0,24)$. Par contre, les individus qui sont sortis des couronnes pour le reste du pays ont un score nettement inférieur $(0,07)$.
On peut dès lors supposer que, pour les individus habitant le centre de Bruxelles en 1995, il faut être bien positionné dans le continuum social afin d'aspirer à le quitter, et donc d'effectuer une trajectoire résidentielle ascendante. D'un côté, dans les couronnes de banlieues, les individus en haut de l'échelle socioéconomique ont des déplacements associés au fait d'y rester ou de gagner le centre, tandis que les groupes moins bien positionnés ont eu plus tendance à déménager vers le reste du pays. II semble également y avoir une différence claire entre ces deux types de mouvements résidentiels. Tandis que le pourcentage de personnes qui déménagent des 


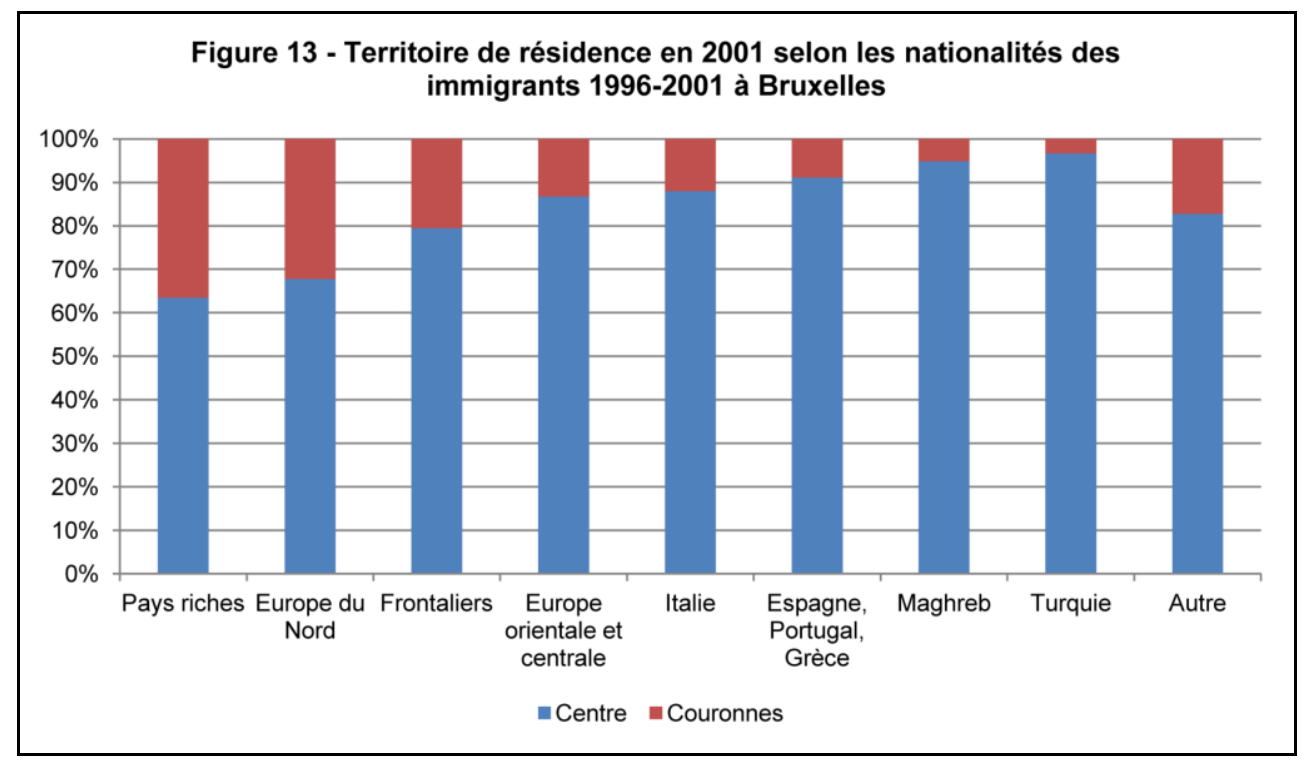

couronnes vers le reste du pays décline graduellement lorsque l'on monte vers les groupes les mieux positionnés, ceci n'est pas le cas pour les déplacements résidentiels vers le centre, où les groupes aux extrêmes du continuum social sont surreprésentés (figure 12). Cela, contrairement à Luxembourg, semble signifier qu'il s'agit à Bruxelles d'un double mouvement. Les gens bien positionnés, peu importe leur lieu de résidence, ont l'opportunité de gagner le centre mais les gens au bas de l'échelle sociale ne peuvent pas accéder aux couronnes périurbaines. Ces derniers peuvent alors se déplacer vers les quartiers centraux plus pauvres et non pas vers l'extérieur de l'agglomération comme dans le cas de Luxembourg.

En ce qui concerne les flux entrant dans l'agglomération bruxelloise, leur profil est plus élevé, aussi bien pour le centre que pour les couronnes, ce qui montre que la plupart des personnes qui s'installent dans l'agglomération sont bien positionnées sur l'échelle sociale. Le rôle de l'immigration est quant à lui moins clair à Bruxelles. Cela est principalement dû au fait que $96 \%$ des immigrants sont rapportés comme «missings » en 200 I. En considérant les pays d'origine, on constate que les immigrants venus des pays liés à la migration ouvrière des années 1970 (Italie, Grèce, Espagne et Portugal, et ensuite Maroc et Turquie) ont eu davantage tendance à s'installer au centre; tandis que pour les immigrants des pays riches (du nord), les couronnes ont absorbé une partie non négligeable de ces flux. Cela suggère que l'immigration internationale semble confirmer et même renforcer les structures d'inégalités existantes, et non comme à Luxembourg à structurer des territoires favorisés.

Ainsi, globalement, les structures d’inégalités semblent relativement stables dans les deux agglomérations, voire même se renforcent durant la période d'observation. Le cas luxembourgeois se distingue par une polarisation classique du bas et du haut de l'échelle sociale au centre. Le cas bruxellois est différent avec une plus forte et plus exclusive polarisation du bas de l'échelle sociale au centre. Nous observons dans les deux agglomérations que les individus mobiles sont nettement plus favorisés que la population stable. Les mobilités résidentielles à Luxembourg concernent les groupes les mieux positionnés, mouvements observés d'abord au centre vers les banlieues. Les flux d'immigration entrants renforcent cette dynamique sociale dictée par les groupes favorisés, où la périphérie constitue une destination privilégiée des immigrants. Contrairement à Luxembourg, l'agglomération de Bruxelles semble marquée par un double mouvement. Les populations les plus favorisées, peu importe leur lieu de résidence, semblent pouvoir gagner le centre, alors que celles du bas de l'échelle semblent ne pas pouvoir accéder aux couronnes périurbaines. Le rôle de l'immigration dans l'évolution des inégalités dans l'agglomération bruxelloise semble moins clair, même si le centre plus précarisé s'avère concerné. 


\section{MODĖLES PROBABILISTES POUR EXPLORER LES STRUCTURES D'INÉGALITÉS}

Afin de comprendre le rôle des différentes trajectoires résidentielles dans les deux agglomérations, nous avons réalisé des analyses de régression logistique binomiales exploratoires (voir tableaux de résultats en annexe) de manière à mettre en lumière, toutes choses étant égales par ailleurs, certains facteurs sociaux et spatiaux susceptibles d'influencer les déplacements observés tout en isolant les éventuels effets de structure. Pour ce faire, une procédure en deux étapes a été établie.

La première consiste en la comparaison de la population ayant effectué un déplacement résidentiel $(n=52161$ à Luxembourg et $n=529710$ à Bruxelles) avec l'ensemble de la population des agglomérations de Luxembourg (1995-200I) et de Bruxelles (1996200I). La deuxième étape consiste à prendre uniquement en considération les personnes ayant effectué un déplacement durant cette période, pour comparer chacune des trajectoires résidentielles discutées plus haut à ces individus s'étant donc déplacés.

Pour les centres des agglomérations de Luxembourg et de Bruxelles, les calculs de probabilités suivants ont été effectués (variable dépendante représentant à chaque fois un mouvement résidentiel donné) à l'aide de quatre/cinq modèles complémentaires :

I) déplacement dans le centre (Bruxelles seulement);

2) déplacement sortant du centre vers les couronnes;

3) déplacement sortant du centre vers le reste du pays;

4) déplacement entrant au centre provenant des couronnes;

5) déplacement entrant au centre en provenance du reste du pays.

Pour les couronnes, les calculs suivants de probabilités ont été effectués (variable dépendante) :

I) déplacement dans les couronnes (Bruxelles seulement);

2) déplacement sortant des couronnes vers le centre;
3) déplacement sortant des couronnes vers le reste du pays;

4) déplacement entrant dans les couronnes provenant du centre;

5) déplacement entrant dans les couronnes en provenance du reste du pays.

Des modèles spécifiques aux individus ayant immigré ont été construits de manière indépendante puisqu'ils n'étaient pas dans les bases de données au premier temps d'observation. Les trajectoires explorées (variables dépendantes) concernant l'immigration sont les suivantes :

I) immigration dans le centre;

2) immigration dans les couronnes.

La liste des variables indépendantes dans les recensements luxembourgeois et belge est la suivante (en date de 200I) : I) groupe social d'appartenance; 2) genre; 3) âge; 4) nationalité; 5) type de ménage; 6) type de logement; 7) date de construction du logement; 8) distance vers le lieux de travail (Bruxelles) et temps de la navette (Luxembourg); 9) moyen de transport utilisé pour se rendre au travail; 10) type de quartier en 1995 (Bruxelles) et en 1996 (Luxembourg) selon la typologie (figures 3 et 4).

\section{I Sédentarité et mobilités au sein des agglomérations (cf. annexes I et 2)}

La mobilité résidentielle au sein de l'agglomération de Luxembourg est peu probable d'être le fait d'un groupe social précarisé. Aucune différence significative n'est visible pour les groupes au haut du continuum social. II est aussi plus probable que ce soit des individus de nationalité inconnue ou apatride $(I, 2$, à lire comme une probabilité) que des Luxembourgeois. II s'agit également de probabilités associées aux classes d'individus plus âgés (plus de 50 ans) (2,6 pour les 50 à 64 ans, 4,3 pour les 65 à 74 ans et 5,2 pour les $75+$ ans), en couple et localisés dans des communes très défavorisées $(I, 4)$ ou défavorisées. II s'agit enfin d'individus résidant dans d'autres types d'habitat $(I, 3)$ que la maison unifamiliale et l'appartement. La grande sédentarité des "nationaux » et de la population des nationalités des pays frontaliers dans l'agglomération avait par ailleurs été observée au niveau du pays (Lord et Gerber, 20I2). La mobilité résidentielle « nationale », associée à l'étalement urbain, était plutôt en lien avec des groupes sociaux mieux positionnés, ce qui ne semble pas être le cas ici pour le territoire spécifique de l'agglomération. 
Dans le cas de Bruxelles, ce sont les groupes les plus précarisés $(0,9)$ qui ont moins de probabilité d'être sédentaires. Pourtant, ce sont bien les nationalités liées à l'immigration ouvrière (Maghreb $(I, I)$, Turquie $(I, 9)$, Europe du Sud $(I, I)$ et naturalisé $(\mathrm{I}, \mathrm{I}))$ qui ont plus de chance d'être sédentaires pour la période 1996-200I. Dans le cas de la population turque, ce fait est peut-être lié à sa concentration dans certains quartiers du centre. À Bruxelles, l'observation des déplacements internes au centre est possible. Les mobilités résidentielles qui concernent le centre montrent que ce sont les « groupes inférieurs $(I, I)$, les 'missing' $(I, 3)$, et les nationalités » liées à l'immigration ouvrière (Maghreb, Turquie, Europe du Sud et naturalisé) qui sont concernés par le déménagement à l'intérieur du centre. Ces déplacements concernent des groupes sociaux déjà surreprésentés dans ces secteurs, groupes qui pourraient ne pas avoir l'opportunité de gagner les milieux plus favorisés en couronnes. En ce qui a trait aux déplacements internes dans les couronnes, ces derniers impliquent plus de probabilités pour les groupes au centre du continuum social $(1,2)$ mais également des individus plus jeunes $(I, 3)$ et des « Eurocrates » $(I, 9)$.

\subsection{Entrer au centre des agglomérations de Luxembourg et Bruxelles (cf. annexes I et 2)}

Les trajectoires associées à l'entrée au centre des deux agglomérations pour les périodes considérées sont associées à des dynamiques relativement différenciées. Tout comme à Bruxelles, l'entrée au centre de Luxembourg, en excluant l'immigration, répond à une même dynamique nourrie par nos deux territoires d'origine pris en considération: les couronnes et le reste du pays. Un déménagement en provenance de ces deux origines vers le centre de l'agglomération est associé à de plus fortes probabilités d'appartenir à un profil de «travailleur métropolitain ». Les célibataires ( 1,7 pour les originaires des couronnes, 2,5 pour le reste du pays) ou les couples sans enfants (respectivement I,8 et I,7) des groupes les mieux positionnés $(1,2)$ sur le continuum social sont les plus probables. Ceux-ci proviennent de communes à la fois «très favorisées » ( 5,8 pour les originaires des couronnes) et «très défavorisées ॥ (3,6 pour le reste du pays) et investissent plutôt un type de logement relativement ancien. Les individus qui entrent au centre de l'agglomération grand-ducale possèdent plus de probabilité d'appartenir à des nationalités étrangères, notamment des pays du nord $(9,2)$ ou de nationalités déjà présentes dans le pays (ex. Portugal $(I, 9))$. Dans tous les cas, les trajectoires entrantes au centre de Luxembourg sont associées aux groupes les mieux positionnés. Une analyse plus détaillée par quartier serait souhaitable afin de mieux saisir les éventuelles dynamiques ségrégatives locales, en particulier pour des groupes d'étrangers établis dans le pays depuis plusieurs décennies mais aussi selon le type de commune d'où proviennent les mouvements résidentiels (communes «très défavorisées » et « très favorisées »).

Les trajectoires vers le centre de l'agglomération de Bruxelles montrent quant à elles des probabilités plus importantes pour les groupes au haut de l'échelle sociale, aussi bien pour ceux provenant des couronnes $(I, 2)$ que pour ceux arrivant du reste du pays $(I, 8)$. Les individus qui y déménagent ont un profil jeune $(I, 4$ pour les originaires des couronnes, I,7 pour le reste du pays), célibataire (respectivement $I, I$ et $I, 4$ ) et appartenant à un couple sans enfants $(I, \mid$ et $I, 3)$. $\grave{A}$ partir des couronnes, ce sont les nationalités italienne $(1,4)$ et de l'Europe du Nord $(1,4)$ qui ont plus de probabilité de déménager, tandis que, pour le reste du pays, les Turcs $(2,0)$ et les « autres nationalités » $(I, 7)$ dominent. Notons que ces déplacements ne se dirigent pas vers les quartiers défavorisés, étant donné les plus fortes probabilités associées aux logements plus récents en opposition au patrimoine plus ancien au centre. Ceci est certainement le cas pour ceux venant des couronnes, d'où leur provenance de quartiers "(très) favorisés » $(\mid 4,2)$, et dans une moindre mesure pour ceux provenant du reste du pays originaires de quartiers « défavorisés 》 $(1,6)$. Peut-on, comme à Luxembourg, envisager que pour les gens arrivant du reste du pays, un tel déménagement vers la capitale signifie une ascension sociale? Du moins, pour les individus provenant des couronnes, ce sont plutôt des parcours associés à des personnes plus aisées qui gagnent, peut-être temporairement, le centre.

\subsection{Sortir du centre des agglomérations de Luxembourg et Bruxelles}

Si le centre comme pôle d'activité montre une certaine attraction pour les profils « jeunes travailleurs métropolitains », il l'est peut-être un peu moins pour les travailleurs plus âgés. Les individus de ces groupes sortent des quartiers "défavorisés 》 et gagnent des logements plus récents en périphérie. II s'agit de couples sans enfants $(I, I)$ qui continuent à travailler dans le centre, comme en témoigne la distance vers le lieu de travail (I,7 pour la catégorie $20-40 \mathrm{~km})$. Les nationalités des «Eurocrates » $(I, 8)$ et des (autres) pays riches $(I, 6)$ sont ici concernées. Concernant les 
probabilités associées à ces trajectoires sortantes, on note une nette sous-représentation des groupes du bas du continuum social $(0,8)$, notamment des Turcs $(0,3)$ et des Maghrébins $(0,3)$, qui, on en fait l'hypothèse, n'ont pas les moyens d'accéder à ce mouvement ascendant en termes d'environnement résidentiel. Au Luxembourg, on constate des tendances fortement similaires, mais essentiellement tournées vers la périurbanisation. Ces trajectoires sortantes, associées à la classe supérieure $(I, 4)$, concernent des individus de nationalités des pays frontaliers $(I, 7)$ tout comme des Portugais $(1,8)$ positionnés tout en haut du continuum social. II s'agit, globalement, d'individus possédant les moyens pour accéder aux prix particulièrement élevés des logements de la périphérie. Ces mouvements résidentiels sont significativement associés à des étrangers déjà présents dans le pays en comparaison des Luxembourgeois.

De manière saillante, les trajectoires résidentielles au sein des couronnes, ainsi que celles du centre ou des couronnes vers le reste du pays, donc à l'extérieur du territoire de l'agglomération, ont beaucoup plus de probabilités de concerner les groupes sociaux les moins bien positionnés, tant à Bruxelles qu'à Luxembourg. Certaines différences sont cependant visibles. Dans les deux agglomérations, ces trajectoires résidentielles impliquent davantage de probabilités de déménager dans un logement plutôt âgé, mais aussi dans des communes/quartiers "défavorisés », voire " très défavorisés », ce qui suggère un déplacement résidentiel descendant en matière d'environnement résidentiel. Cette dynamique semble particulièrement présente au Luxembourg, où les étrangers de nationalités différentes des pays frontaliers, portugaise et des pays du Nord, montrent des probabilités nettement plus importantes de sortir de l'agglomération ou des couronnes pour se diriger vers le reste du pays, tout particulièrement vers l'ancien bassin minier en désindustrialisation. Dans le cas de Luxembourg, ces mouvements résidentiels suggèrent, sinon un embourgeoisement, du moins une amélioration de la position socioéconomique du centre, et surtout des couronnes périurbaines. Avec une dynamique similaire, dans le cas de Bruxelles, la question des pressions immobilières sur les groupes défavorisés qui n'ont pas les moyens de déménager dans les couronnes proches peut être posée, tout comme une autre sur les affinités socioculturelles au sein des quartiers précarisés du centre.

\subsection{Immigrer dans les agglomérations de Luxembourg et Bruxelles}

La double migration, constituée de groupes sociaux situés aux deux extrêmes du continuum social, est fortement représentée au niveau du centre des deux agglomérations européennes. Dans le cas de Luxembourg, les groupes sociaux au haut $(1,6)$ de l'échelle sociale montrent des probabilités plus élevées d'entrer au centre, un territoire central pouvant faire office de lieu d'arrivée et/ou de passage avant l'intégration des territoires dits « nationaux ».

Cette même double migration relève d'une géographie très claire pour l'agglomération de Bruxelles. Même si les groupes sociaux ne sont pas disponibles comme à Luxembourg, à cause d'un manque d'effectifs, les probabilités selon les nationalités montrent clairement que les groupes issus de l'immigration ouvrière ont significativement plus de probabilités d'immigrer au centre, tandis que les pays considérés comme plus riches (Europe du Nord, pays Frontaliers, USA, Canada, Japon) (de I,5 à 2,2) ont plutôt tendance à s'installer dans un logement récent $(2,2)$ dans les couronnes de l'agglomération.

\section{DISCUSSION CONCLUSIVE}

L'analyse des trajectoires résidentielles par groupes sociaux dans les agglomérations de Bruxelles et Luxembourg montre que les libertés de mouvements résidentiels ne sont pas partagées par tous les groupes sociaux. Certes, nos observations tendent vers des patterns classiques, comme celles des « jeunes travailleurs métropolitains » au début de leur parcours résidentiel s'établissant au centre. Une dynamique attendue était celle de la périurbanisation des groupes situés en-haut du continuum social, observée à Bruxelles et nettement moins à Luxembourg. Tous les groupes sociaux n'ont en effet pas les moyens de gagner la périphérie favorisée. Ainsi, les groupes en bas du continuum social ont plutôt tendance à rester dans le centre de Bruxelles. Certains groupes semblent échapper à cette logique, en déménageant notamment vers le reste du pays, où ils s'établissent dans des logements plus ou moins vétustes. Les Turcs et les Maghrébins, quant à eux, ne sont pas concernés par ce mouvement. Dans les couronnes, par contre, les moins fortunés semblent repoussés vers l'extérieur des deux agglomérations. $\mathrm{Si}$ les couronnes gagnent alors en habitants, c'est grâce à des mouvements provenant essentiellement du centre, contrairement à Luxembourg où l'immigration "dorée » joue un rôle de premier plan dans la périurbanisation. 
À Bruxelles, c'est le centre qui semble nourri par l'immigration. L'analyse des trajectoires immigrantes montre qu'elles se greffent sur les structures sociospatiales existantes. Même si, en nombre absolu, la plupart des immigrants venus des pays dits riches s'établissent dans le centre de l'agglomération, ils sont néanmoins surreprésentés dans les couronnes. II peut être ici envisagé que ce groupe possède les moyens de se loger selon ses aspirations : au centre comme jeune travailleur urbain, ou dans la périphérie pour les familles plus aisées. Les immigrants dits ouvriers semblent ne pouvoir accéder qu'aux quartiers centraux. Au Luxembourg, le centre reste attractif pour les immigrants des deux extrêmes du continuum social qui y sont surreprésentés, tandis que les groupes moyens s'orientent plutôt vers les couronnes. $\mathrm{Ce}$ territoire n'est guère accessible pour les moins favorisés, ces derniers s'installant dans le centre, mais étant surtout surreprésentés dans le reste du pays.

Nos observations semblent aller dans le même sens que celles de Donzelot et Jaillet (1997) sur les effets de la mondialisation venant renforcer les structures d'inégalités, en court-circuitant les transitions résidentielles fordistes. Mais elles montrent aussi l'importance des structures d'inégalités existantes et de l'inertie des contextes structurels historiques. La suburbanisation des classes moyennes et l'immigration ouvrière des années 1960-70 ont eu comme conséquence l'appauvrissement du centre à Bruxelles, avec la structuration des couronnes périurbaines plus riches. Dans le cas de l'agglomération de Luxembourg, son centre et les couronnes adjacentes sont plutôt bien positionnés à cause de facteurs liés à l'augmentation de la migration "dorée », groupes sociaux cherchant sans doute davantage un environnement résidentiel plus urbain que rural. Cela dit, tant à Luxembourg que Bruxelles, l'immigration confirme et même renforce les structures d'inégalités existantes, ratant d'autant l'occasion, avec une migration diversifiée, d'améliorer la mixité spatiale et sociale des deux agglomérations.

Notre analyse comparative a voulu clarifier les grandes tendances et les effets des trajectoires résidentielles des différents groupes sociaux sur les structures d'inégalités de deux agglomérations européennes fortement affectées par la mobilité résidentielle et l'immigration internationale. En distinguant le centre des agglomérations et leurs couronnes périurbaines, notre analyse a pu montrer trois constats relativement clairs: I) les groupes en bas de l'échelle sociale sont limités en ce qui concerne leurs possibilités de déménager; 2) l'ensemble des trajectoires observées s'aligne sur les structures d'inégalités existantes; 3) l'immigration internationale vient confirmer et renforcer les structures existantes. Les territoires des agglomérations luxembourgeoise et bruxelloise ressortent donc comme des espaces historiquement inégalitaires, où l'inscription spatiale et sociale de l'immigration internationale se voit relativement prescrite en matière d'espaces résidentiels.

Enfin, le choix d'utiliser la division fonctionnelle entre le centre et les couronnes pour l'ensemble des agglomérations ainsi que l'échelle communale comme unité d'analyse, de manière à avoir des délimitations objectives et non liées aux groupes sociaux, ont pour conséquence une perte significative de la complexité spatiale et sociale. Les processus de concentration résidentielle se moyennent assurément au niveau communal. En ce sens, et pour compléter nos analyses exploratoires, il serait pertinent de croiser les modèles probabilistes, que nous avons pu développer dans le cadre de cet article, avec des analyses multiniveaux (Goldstein, 2003) pouvant raisonner à différentes échelles spatiales complémentaires. Cellesci permettraient en outre d'améliorer la prise en compte d'unités spatiales plus fines pour que les flux entrants et sortants puissent également faire émerger des processus liés à la gentrification ou la ségrégation. De telles analyses ouvriraient également la possibilité de cibler certains secteurs urbains pour y mener des analyses plus qualitatives, notamment sur les parcours intégratifs des immigrants plus ou moins bien positionnés.

\section{BIBLIOGRAPHIE}

ATKINSON, A.B. et E. MARLIER (2010, eds.). Income and living conditions in Europe, Luxembourg, Publications Office of the European Union.

BAUMAN, Z. (2000). Liquid modernity, Oxford, Blackwell.

BESCH, S. (2006). « Immigration et migrations transfrontalières », Dimensions socioéconomiques de la mobilité transfrontalière, Actes du séminaire transfrontalier EURES-OIE, I4-I5 mars 2005, Luxembourg, P. I5-2I.

BOURDIEU, P. (1979). La distinction, critique sociale du jugement, Paris, Éditions de Minuit.

CARPENTIER, S. (20I0, dir.). La mobilité résidentielle transfrontalière entre le Luxembourg et ses régions voisines, Luxembourg, Editions Saint-Paul. 
CORIJN, E., C. VANDERMOTTEN, J.M. DECROLY et E. SWYNGEDOUW (2009). «États-généraux de Bruxelles. Bruxelles, ville internationale », Brussels Studies, note de synthèse no $13,12 \mathrm{p}$.

D'ANDRIMONT, C., B. WAYENS, G. VAN HAMME et A. ROMAINVILLE (2007). Localisation et délocalisation d'entreprises en Région bruxelloise, rapport inédit pour EUDIP two, Bruxelles, IGEAT-ULB, I04 p.

DEBOOSSERE, P., T. EGGERICKX, E. VAN HECKE et B. WAYENS (2009). «États-généraux de Bruxelles. La population bruxelloise : un éclairage démographique » Brussels Studies, note de synthèse no $3,18 p$.

DUREAU, F. (2002). « Les systèmes résidentiels : concepts et applications », In LÉVY, J.-P. et F. DUREAU (dir.), L'accès à la ville. Les mobilités spatiales en question, Paris, L'Harmattan, p. 355382.

EGGERICKX, T., C. KESTELOOT, P. GERBER, C. SOHN, F. DE MAESSCHALCK, S. LORD et J.-P. HERMIA (2009). Individual economic insecurity factors: A dynamic typology of socio-demographic inequalities on the neighbourhood level. Spatial application for Belgium and Luxembourg from 1991 to 2006, papier présenté lors du XXVI IUSSP International Population Conference, 27 septembre - 2 octobre, Marrakech.

FEHLEN, F. (2009). « L'immigration dorée », In BOUSCH, P., P. GERBER et T. CHILLA (dir.), Der Luxembourg Atlas / Atlas du Luxembourg, Cologne, Éditions Emons, p. I70-I7I.

FITOUSSI, J.-P., E. LAURENT et J. MAURICE (2004). Ségrégation urbaine et intégration sociale, Paris, La Documentation française, $327 \mathrm{p}$.

FLEURY, C. (2009). « La mosaïque luxembourgeoise », Vivre au Luxembourg, vol. 54, Luxembourg, CEPS/INSTEAD, 2 p.

GERBER, P. et M. PRUVOT (2005). « La ville de Luxembourg, mosaïque socio-résidentielle », Population \& Territoire, vol. 7, Luxembourg, CEPS/INSTEAD, 20 p.
GERBER, P., S. CARPENTIER, S. PETIT et I. PIROTH (2008). « Mobilités quotidienne et résidentielle : un aperçu à travers l'outil MobilluxWeb », Population \& Territoire, vol. 13, Luxembourg, CEPS/INSTEAD, $20 \mathrm{p}$

GERBER, P., O. KLEIN et S. CARPENTIER (20I2). « Local mobilities and cross-border urban sprawl », In SOHN, C. (dir.), Luxembourg: An Emerging Cross-Border Metropolitan Region, Bruxelles, Peter Lang, p. I4I-I59.

GERBER, P. et I. PIGERON-PIROTH (2004). « Les villes de Luxembourg et d'Esch/Alzette : quelques aspects économiques et géographiques à la lumière du recensement de 2001 ॥. Population \& Territoire, vol. 4, Luxembourg, CEPS/INSTEAD, 12 p.

GERBER, P. et I. PIGERON-PIROTH (2009). « Ein Porträt der Vielfalt: Ausländer in Luxemburg und Esch », In BOUSCH, P., P. GERBER et T. CHILLA (dir.), Der Luxembourg Atlas / Atlas du Luxembourg. Cologne, Editions Emons, p. I74- I75.

GOLDSTEIN, H. (2003). Multilevel Statistical Models. Londres, Arnold Publishers, 243 p.

HARTMANN-HIRSCH, C. (2007a). « Les étrangers et le marché de l'emploi : politiques migratoires et immigration », Cahier PSELL, vol. I57, Luxembourg, CEPS/INSTEAD, 70 p.

HARTMANN-HIRSCH, C. (2007b). Les réalités des politiques migratoires et leur perception dans un petit État-nation, Rapport final pour FNR-VIVRE, Luxembourg, CEPS/INSTEAD.

HERMIA, J.-P., T. EGGERICKX, C. KESTELOOT, P. GERBER, C. SOHN, F. DE MAESSCHALCK et S. LORD (2009). Facteurs de précarité au niveau individuel : une typologie dynamique des inégalités sociodémographiques à l'échelle du quartier. Application dans l'espace aux cas belge et luxembourgeois pour la période 1991-2006, papier présenté lors du XXVI IUSSP International Population Conference, 27 septembre - 2 octobre, Marrakech.

KAUFMANN, V. (2000). Mobilité quotidienne et dynamiques urbaines - La question du report modal, Lausanne, Presses polytechniques et universitaires romandes. 
KAUFMANN, V. (2005). « Mobilités et réversibilités : vers des sociétés plus fluides ? », Cahiers internationaux de sociologie, vol. I 18, Paris, Presses universitaires de France, p. I 19-135.

KESTELOOT, C. (1999) « De la ségrégation à la division: l'évolution et les enjeux futurs de la structure socio-spatiale bruxelloise ». In WITTE, E. et al. (eds.) Het statuut van Brussel, Brussel: Larcier, p. 155-189.

KESTELOOT, C., K. SLEGERS, L. VANDEN BROUCKE, C. VANDERMOTTEN, P. MARISSAL, G. VAN HAMME, B. IPPERSEEL, R. NAIKEN et S. DE BETHUNE (2006). Atlas van achtergestelde buurten in moeilijkheden in Belgische stadsgewesten, Brussel: FOD Maatschappelijke integratie, Grootstedenbeleid.

LANGERS, J. (2005). « Potentiel de croissance économique et démographie, Projection 20052055 », Bulletin du Statec, vol. 4, Luxembourg, STATEC.

LORD, S. et P. GERBER (2009). « Immigration et intégration: Trajectoires résidentielles (inter)nationales et dynamiques ségrégatives locales au Luxembourg », Espace Populations Sociétés, vol. 2009, no I, p. 85-103.

LORD, S. et P. GERBER (20I2). « Residential and Cross-border Mobility : A catalyst of Social Polarisation? ॥ In SOHN, C. (ed.), Luxembourg: an emerging cross-border metropolitan region, Brussels: Peter Lang, p. 161-184.

LORD, S. et P. GERBER (20I3). « Immigration, dynamiques socioéconomiques territoirales et movements résidentiels. Quelles perspectives pour les residents du Luxembourg? ", Annales de Géographie, n690, mars-avril, p. 175-199.

LORD, S., P. GERBER, C. SOHN, T. EGGERICKX, J.P. HERMIA, T. CASSIERS, F. DE MAESSCHALCK et C. KESTELOOT (20I I). « Temporal and spatial analysis of social inequalities: An innovative method to grasp social inequalities evolution on the territory ॥, Working Paper, vol. 47, Luxembourg, CEPS/INSTEAD, 40 p.

LUYTEN, S. et E. VAN HECKE (2007). « De Belgische stadsgewesten $200 \mathrm{I}$ ॥, Statistics Belgium - Working papers, vol. 14, Bruxelles, Direction générale Statistique et Information économique, $80 \mathrm{p}$.
MAURIN, E. (2004). Le ghetto français : Enquête sur le séparatisme social, Paris, Éditions du Seuil et la République des Idées, 96 p.

MONTEBELLO, F. (200I, dir.). « Un siècle d'immigration au Luxembourg », Passerelles, vol. 22, Luxembourg, CLAE, 255 p.

PRIES, P. (1999). Migration and Transnational Spaces, Aldershot, Ashgate.

SASSEN, S. (199I). The global city: New York, London, Tokyo, Princeton, Princeton University Press.

SCHMITZ, F. et P. GERBER (20II). "Voiture ou transports en commun? Comment les frontaliers se rendent-ils au travail en 2010 ? „ Vivre au Luxembourg, vol. 78, Luxembourg, CEPS/INSTEAD, 2 p.

SOHN, C., B. REITEL et O. WALTHER (2009). « Cross-border metropolitan integration in Europe: The case of Luxembourg, Basel, and Geneva », Environment and Planning C: Government and Policy, vol. 27, no 5, p. 922-939.

VANDERMOTTEN, C., E. LECLERCQ, T. CASSIERS et B. WAYENS (2009). «États-généraux de Bruxelles. L'économie bruxelloise » Brussels Studies, note de synthèse no $7,14 \mathrm{p}$.

WEISS, A. (2005). « The transnationalization of social inequality: Conceptualizing social positions on a world scale », Current Sociology, vol. 53, no 4, p. 707-728.

WILLAERT, D. (2010). « De recente internationalisering van het Brussels gewest en de Vlaamse rand ॥, Interface demography. Working papers, vol. 2010-2, Bruxelles, VUB 21 p.

VAN CRIECKINGEN, M. (2009). « Moving In/Out of Brussels' Historical Core in the Early 2000s: Migration and the Effects of Gentrification "), Urban Studies, vol. 46, no 4, p. 825-848.

VAN HAMME, G., Y. WERTZ et V. BIOT (20I I). « La croissance économique sans le progrès social. L'état de lieu à Bruxelles » Brussels Studies, vol. 48, 21 p. 
EUE • Une mise à l'épreuve de politiques énergétiques tunisiennes • c-2 I

\begin{tabular}{|c|c|c|c|c|c|c|c|c|c|c|}
\hline & $\begin{array}{l}\text { Déména- } \\
\text { gement }\end{array}$ & $\begin{array}{c}\text { Mobilité du } \\
\text { centre vers les } \\
\text { couronnes }\end{array}$ & $\begin{array}{c}\text { Mobilité du } \\
\text { centre vers le } \\
\text { reste du pays }\end{array}$ & $\begin{array}{c}\text { Mobilité des } \\
\text { couronnes vers } \\
\text { les centre }\end{array}$ & $\begin{array}{c}\text { Mobilité sortant } \\
\text { des couronnes } \\
\text { vers le reste du } \\
\text { pays }\end{array}$ & $\begin{array}{c}\text { Mobilité entrant } \\
\text { au centre } \\
\text { provenanat du } \\
\text { reste du pays }\end{array}$ & \begin{tabular}{|c|} 
Mobilité entrant \\
dans les \\
couronnes \\
provenant du \\
reste du pays \\
\end{tabular} & \begin{tabular}{|c} 
Immigration \\
dans le centre
\end{tabular} & $\begin{array}{c}\text { Immigration } \\
\text { dans les } \\
\text { couronnes }\end{array}$ & $\begin{array}{l}\text { Immigration } \\
\text { dans le reste di } \\
\text { pays }\end{array}$ \\
\hline & $\exp (b)$ & $\exp (b)$ & $\exp (b)$ & $\exp (b)$ & $\exp (b)$ & $\exp (b)$ & $\exp (b)$ & $\begin{array}{l}\exp (b) \\
\end{array}$ & \begin{tabular}{|l|l|}
$\exp (b)$ \\
\end{tabular} & $\exp (b)$ \\
\hline \begin{tabular}{|l} 
Groupes sociaux \\
L_Missings \\
L_G1-3 \\
L_G4-6 \\
L_G7-9 \\
L_G10-12 \\
L_G13-15 \\
L_G16-17 \\
\end{tabular} & $\begin{array}{l}1,02 \bullet \\
0,72 \\
1,10 \\
0,96 \\
0,99 \\
\text { Ref } \\
0,97\end{array}$ & $\begin{array}{l}0,70 \\
0,68 \\
0,62 \\
0,55 \\
0,68 \\
\text { Ref } \\
1,40 \\
\end{array}$ & $\begin{array}{l}1,60 \\
2,63 \\
2,04 \\
1,56 \\
1,33 \\
\text { Ref } \\
0,89 \\
0,\end{array}$ & $\begin{array}{l}0,65 \\
0,42 \\
0,42 \\
0,48 \\
0,81 \\
\text { Ref } \\
1,18\end{array}$ & $\begin{array}{l}0,92 \bullet \\
1,08: \\
1,11: \\
1,20 \\
0,98 \\
\text { Ref } \\
0,66\end{array}$ & $\begin{array}{ll}0,86 & \bullet \\
0,56 & \\
0,45 & \\
0,92 & \bullet \\
0,67 & \bullet \\
\text { Ref } & \\
1,04 & \bullet\end{array}$ & $\begin{array}{l}1,24 \bullet \\
0,65 \\
0,82 \bullet \\
0,92 \bullet \\
1,12 \bullet \\
\text { Ref } \\
1,07 \bullet \\
\end{array}$ & $\begin{array}{l}1,33 \\
0,56 \\
0,66 \\
0,87 \\
0,75 \\
\text { Ref } \\
1,56 \\
\end{array}$ & $\begin{array}{r}0,98: \\
0,97: \\
0,95: \\
1,16 \\
\mathbf{1 , 2 6} \\
\text { Ref } \\
1,15 \\
\end{array}$ & $\begin{array}{l}0,80 \\
1,63 \\
1,48 \\
1,03 \\
1,06 \\
\text { Ref } \\
0,50\end{array}$ \\
\hline \begin{tabular}{|l|} 
Genre \\
Homme \\
Femme \\
\end{tabular} & $\begin{array}{l}1,03 \bullet \\
\text { Ref }\end{array}$ & $\begin{array}{l}1,11 \bullet \\
\text { Ref }\end{array}$ & $\begin{array}{l}1,05 \bullet \\
\text { Ref }\end{array}$ & $\begin{array}{l}1,14 \bullet \\
\text { Ref }\end{array}$ & $\begin{array}{l}0,88 \\
\text { Ref }\end{array}$ & $\begin{array}{l}1,02 \bullet \\
\text { Ref }\end{array}$ & $\begin{array}{l}1,10 \bullet \\
\text { Ref }\end{array}$ & $\begin{array}{l}1,21 \\
\text { Ref }\end{array}$ & $\begin{array}{l}0,92 \\
\text { Ref }\end{array}$ & $\begin{array}{l}0,86 \\
\text { Ref }\end{array}$ \\
\hline \begin{tabular}{|l|} 
Groupes d'âge \\
18 à 24 ans \\
25 à 34 ans \\
35 à 49 ans \\
50 à 64 ans \\
65 à 74 ans \\
75 ans et plus
\end{tabular} & $\begin{array}{l}0,65 \\
0,44 \\
\text { Ref } \\
2,63 \\
4,34 \\
5,18\end{array}$ & $\begin{array}{l}0,77: \bullet \\
0,85 \\
\text { Ref } \\
1,20 \\
0,88 \\
1,16\end{array}$ & $\begin{array}{l}0,89 \bullet \\
1,05: \\
\text { Ref } \\
0,92: \\
0,79 \\
0,91\end{array}$ & $\begin{array}{l}0,87 \bullet \\
0,97: \\
\text { Ref } \\
0,94: \\
0,88: \\
0,53:\end{array}$ & $\begin{array}{l}0,96 \bullet \\
\mathbf{0}, 82 \\
\text { Ref } \\
0,99 \\
1,27\end{array}$ & $\begin{array}{ll}0,60 & \bullet \\
0,77 & \bullet \\
\text { Ref } & \\
0,27 & \bullet \\
0,38 & \\
0,75 & \bullet\end{array}$ & $\begin{array}{l}1,07 \bullet \\
1,13 \bullet \\
\text { Ref } \\
1,00 \\
0,90 \\
0,98\end{array}$ & $\begin{array}{l}0,67 \\
0,88 \\
\text { Ref } \\
0,75 \\
0,39 \\
0,39\end{array}$ & $\begin{array}{l}0,82 \\
0,88 \\
\text { Ref } \\
0,93\end{array}$ & $\begin{array}{l}1,79 \\
1,32 \\
\text { Ref } \\
1,51 \\
2,02 \\
1,83\end{array}$ \\
\hline 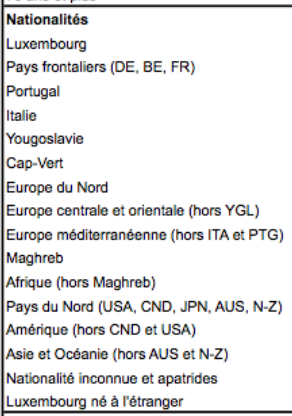 & $\begin{array}{l}\text { Ref } \\
0,66\end{array}$ & $\begin{array}{l}\text { Ref } \\
1,72: \\
1,86 \\
1,81 \\
1,84: \\
2,02: \\
1,66: \\
1,55: \\
1,59: \\
0,00: \\
1,67: \\
0,94: \\
0,54: \\
1,05: \\
0,00 \\
0,83:\end{array}$ & 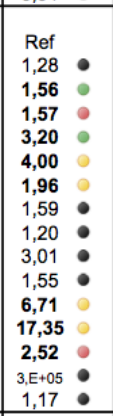 & 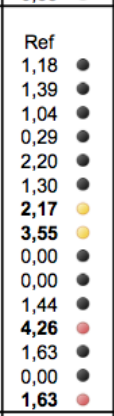 & $\begin{array}{l}\text { Ref } \\
0,75 \\
0,50 \\
1,07 \\
0,97: \\
0,50: \\
0,54 \\
0,80: \\
1,11: \\
0,00: \\
1,21: \\
0,00: \\
0,00: \\
0,73: \\
1,24: \\
0,71\end{array}$ & $\begin{array}{l}\text { Ref } \\
0,90 \\
1,90\end{array}$ & $\begin{array}{ll} & \\
\text { Ref } \\
0,59\end{array}:$ & $\begin{array}{l}\text { Ref } \\
1,41: \\
1,18: \\
2,62: \\
0,18 \\
3,18 \\
1,84 \\
1,09: \\
3,86: \\
1,25: \\
1,28: \\
2,08 \\
3,83: \\
1,37: \\
0,65: \\
1,46\end{array}$ & $\begin{array}{l}\text { Ref } \\
0,77 \\
0,66 \\
0,62 \\
0,55 \\
0,41 \\
1,35 \\
1,24 \\
0,75 \\
0,89 \\
0,77 \\
1,69 \\
0,48 \\
1,43 \\
0,52 \\
0,78\end{array}$ & 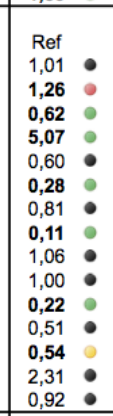 \\
\hline 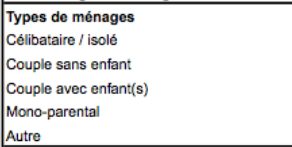 & $\begin{array}{l}\mathbf{0 , 5 1} \\
\mathbf{0 , 5 0} \\
\text { Ref } \\
0,86 \\
0,39 \\
0,\end{array}$ & $\begin{array}{ll}0,53 & \bullet \\
0,83 & \bullet \\
\text { Ref } & \\
0,61 & \bullet \\
0,62 & \bullet\end{array}$ & $\begin{array}{l}1,17 \bullet \\
1,29 \\
\text { Ref } \\
0,95 \\
0,80\end{array}$ & $\begin{array}{l}1,65 \\
1,77 \\
\text { Ref } \\
1,75 \\
1,33 \\
\end{array}$ & $\begin{array}{l}1,13 \bullet \\
1,06 \bullet \\
\text { Ref } \\
0,86 \\
0,81\end{array}$ & $\begin{array}{l}2,45 \\
1,71 \\
\text { Ref } \\
1,60 \\
2,59\end{array}$ & $\begin{array}{l}0,94 \bullet \\
1,18 \bullet \\
\text { Ref } \\
1,08 \\
1,11 \bullet\end{array}$ & $\begin{array}{l}2,46 \\
1,43 \\
\text { Ref } \\
1,45 \\
1,63\end{array}$ & $\begin{array}{l}\mathbf{0 , 4 8} \\
\mathbf{0 , 8 2} \\
\mathrm{Ref} \\
\mathbf{0 , 7 1} \\
1,15\end{array}$ & $\begin{array}{ll}0,76 & \bullet \\
0,88 & \bullet \\
\text { Ref } & \\
1,00 & \bullet \\
0,54 & \bullet\end{array}$ \\
\hline \begin{tabular}{|l}
$\begin{array}{l}\text { Yypes de logements } \\
\text { Maison individuelle } \\
\text { Immeuble collecifif } \\
\text { Autre }\end{array}$ \\
\end{tabular} & $\begin{array}{l}\text { Ref } \\
0,61 \text {. } \\
1,30\end{array}$ & $\begin{array}{l}\text { Ref } \\
0,95- \\
1,16 \bullet\end{array}$ & $\begin{array}{l}\text { Ref } \\
0,99 \text { : } \\
0,82 \text { - }\end{array}$ & $\begin{array}{l}\text { Ref } \\
3,07 \text { : } \\
0,88 \text { - }\end{array}$ & $\begin{array}{l}\text { Ref } \\
1,00 \text { - } \\
0,60\end{array}$ & $\begin{array}{l}\text { Ref } \\
2,65 \text { : } \\
1,30\end{array}$ & $\begin{array}{l}\text { Ref } \\
0,76 \text { : } \\
0,66 \text { - }\end{array}$ & $\begin{array}{l}\text { Ref } \\
2,74 \\
1,05\end{array}$ & \begin{tabular}{|l} 
Ref \\
0,53 \\
0,62
\end{tabular} & $\begin{array}{l}\text { Ref } \\
0,68 \text { - } \\
1,38\end{array}$ \\
\hline \begin{tabular}{|l} 
Année d'achèvement du bátiment \\
Avant 11919 \\
1919-45 \\
$1946-55$ \\
$1956-60$ \\
$1961-70$ \\
$1971-80$ \\
$1981-86$ \\
Aprés 1986 \\
\end{tabular} & $\begin{array}{l}5,05 \\
5,11 \\
5,54 \\
5,05 \\
5,78 \\
5,67 \\
4,10 \\
\operatorname{Ref}\end{array}$ & $\begin{array}{l}0,52 \\
0,53 \\
0,62 \\
1,81 \\
1,59 \\
1,39 \\
1,34 \\
\text { Ref }\end{array}$ & $\begin{array}{l}1,61 \\
1,51 \\
0,93 \\
0,63 \\
0,44 \\
0,81 \\
0,76 \\
\text { Ref }\end{array}$ & \begin{tabular}{|ll}
0,96 & $\bullet$ \\
$\mathbf{2 , 8 3}$ & $\bullet$ \\
$\mathbf{5 , 5 6}$ & $\bullet$ \\
$\mathbf{2 , 2 9}$ & $\bullet$ \\
1,74 & $\circ$ \\
0,81 & $\bullet$ \\
1,02 & $\bullet$ \\
Ref & \\
\end{tabular} & $\begin{array}{l}1,13 \bullet \\
\mathbf{1}, \mathbf{3 0} \\
0,83 \\
\mathbf{0 , 7 0} \\
\mathbf{0 , 5 1} \\
\mathbf{0 , 6 9} \\
1,11 \\
\text { Ref }\end{array}$ & $\begin{array}{l}1,80 \bullet \\
3,14 \\
5,45 \\
3,25\end{array}$ & $\begin{array}{l}\mathbf{0 , 7 0} \\
\mathbf{0 , 5 4} \\
\mathbf{0 , 6 6} \\
\mathbf{1 , 6 1} \\
\mathbf{1 , 5 0} \\
1,21 \\
0,29 \\
\text { Ref }\end{array}$ & $\begin{array}{l}1,45 \\
4,22 \\
6,33 \\
3,14 \\
2,17 \\
1,17 \\
0,83 \\
\text { Ref }\end{array}$ & $\begin{array}{l}\mathbf{0 , 5 3} \\
\mathbf{0 , 2 4} \\
\mathbf{0 , 3 3} \\
\mathbf{0 , 8 0} \\
1,12 \\
1,23 \\
\mathbf{1 , 2 6} \\
\text { Ref }\end{array}$ & 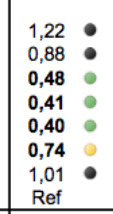 \\
\hline \begin{tabular}{|l} 
Temps de trajet au travail \\
Moins de 15 minutes \\
$15-29$ minutes \\
$30-59$ minutes \\
Plus d'une heure \\
\end{tabular} & $\begin{array}{l}\text { Ref } \\
0,77 \\
0,73 \\
0,76\end{array}$ & $\begin{array}{l}\text { Ref } \\
1,19 \\
0,57 \\
0,25\end{array}$ & $\begin{array}{l}\text { Ref } \\
0,76 \text { : } \\
2,29 \\
4,39\end{array}$ & $\begin{array}{l}\text { Ref } \\
0,61 \text { - } \\
0,19 \\
0,18\end{array}$ & $\begin{array}{l}\text { Ref } \\
1,22 \\
2,21 \\
2,31\end{array}$ & $\begin{array}{l}\text { Ref } \\
0,89 \\
0,33\end{array}$ & $\begin{array}{l}\text { Ref } \\
\mathbf{1 , 2 4} \\
1,03 \\
\mathbf{0 , 5 8}\end{array}$ & $\begin{array}{l}\text { Ref } \\
0,53 \\
0,22 \\
0,20\end{array}$ & $\begin{array}{l}\text { Ref } \\
\mathbf{1 , 8 5} \\
1,01 \\
0,97\end{array}$ & $\begin{array}{l}\text { Ref } \\
0,96:- \\
3,70: \\
4,15\end{array}$ \\
\hline $\begin{array}{l}\text { Moyen de transport pour le travail } \\
\text { Auto } \\
\text { Transport en commun } \\
\text { Transport acif } \\
\text { Autre transport } \\
\end{array}$ & $\begin{array}{l}\text { Ref } \\
1,20 \\
1,90 \\
1,14\end{array}$ & $\begin{array}{l}\text { Ref } \\
1,15: \\
0,71: \\
0,88\end{array}$ & $\begin{array}{l}\text { Ref } \\
0,77 \text { • } \\
0,87 \text { : } \\
0,77 \text {. }\end{array}$ & $\begin{array}{l}\text { Ref } \\
2,04 \\
2,59 \\
1,27\end{array}$ & $\begin{array}{l}\text { Ref } \\
0,64 \\
1,02 ~: \\
0,93:\end{array}$ & $\begin{array}{l}\text { Ref } \\
1,47 \\
3,94 \\
0,73\end{array}$ & $\begin{array}{l}\text { Ref } \\
0,81 \\
0,41 \\
0,84\end{array}$ & $\begin{array}{l}\text { Ref } \\
2,32 \\
2,73 \\
0,75\end{array}$ & $\begin{array}{l}\text { Ref } \\
0,92 \\
0,49 \\
0,65\end{array}$ & $\begin{array}{l} \\
\text { Ref } \\
0,44 \\
0,59 \\
1,62\end{array}$ \\
\hline $\begin{array}{l}\text { Type de commune de travail } \\
\text { Commune rurale et pole secondaire } \\
\text { Périurbain éloigné } \\
\text { Deuxième couronne dagglomération } \\
\text { Premiere couronne d'agglomération } \\
\text { Bassin minier } \\
\text { Ville dense - Luxembourg } \\
\text { Ville dense - Eschl/Alzette } \\
\end{array}$ & $\begin{array}{l}1,00 \bullet \\
0,95 \bullet \\
0,93 \bullet \\
0,90 \\
1,11 \\
\operatorname{Ref} \\
1,12 \bullet \\
\end{array}$ & $\begin{array}{ll}0,12 & \bullet \\
0,20 & \bullet \\
0,83 & \bullet \\
0,94 & \bullet \\
0,13 & \bullet \\
\text { Ref } & \\
0,16 & \\
\end{array}$ & 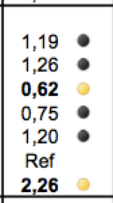 & $\begin{array}{l}0,17 \\
0,49 \\
0,60 \\
1,02 \\
0,19 \\
\text { Ref } \\
0,13\end{array}$ & $\begin{array}{l}0,67 \\
1,73 \\
1,44 \\
0,92 \\
3,01 \\
\text { Ref } \\
2,57\end{array}$ & \begin{tabular}{|ll}
0,16 & \\
0,21 & \\
0,43 & \\
0,59 & \\
0,19 & \\
Ref & \\
0,13 & \\
\end{tabular} & 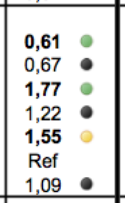 & $\begin{array}{l}0,08 \\
0,22 \\
0,31 \\
0,69 \\
0,04 \\
\text { Ref } \\
0,05\end{array}$ & $\begin{array}{ll}0,13 & \\
0,53 & \\
1,75 & \circ \\
2,19 & \circ \\
0,83 & \bullet \\
\text { Ref } & \\
0,89 & \bullet \\
\end{array}$ & $\begin{array}{c}19,63 \\
4,94 \\
1,52\end{array}$ \\
\hline \begin{tabular}{|l|} 
Type de contrat \\
CDI \\
Fonctionnaire \\
CDD
\end{tabular} & $\begin{array}{l}\text { Ref } \\
0,93 \\
1,07 \\
0,95\end{array}$ & $\begin{array}{l}\text { Ref } \\
1,14: \\
1,03 \\
0,51\end{array}$ & $\begin{array}{l}\text { Ref } \\
1,02 \\
0,87 \\
1,55:\end{array}$ & $\begin{array}{l}\text { Ref } \\
0,66 \\
1,80 \\
2,01\end{array}$ & $\begin{array}{l}\text { Ref } \\
0,72 \\
0,86 \\
1,24\end{array}$ & \begin{tabular}{|l|} 
\\
Ref \\
1,19 \\
1,73 \\
0,34
\end{tabular} & $\begin{array}{l}\text { Ref } \\
1,00 \\
0,80 \\
1,22\end{array}$ & $\begin{array}{l}\text { Ref } \\
1,44 \\
1,43 \\
1,31\end{array}$ & $\begin{array}{l}\text { Ref } \\
0,94 \\
0,90 \\
1,16\end{array}$ & $\begin{array}{ll} & \\
\text { Ref } \\
0,71 & \bullet \\
0,74 & \\
0,56 & \bullet \\
\end{array}$ \\
\hline \begin{tabular}{|l} 
Type de commune en 1995 \\
Commune très déffavorisée \\
Commune defáavorisée \\
Commune moyenne \\
Commune favorisée \\
commune trés favorisée
\end{tabular} & $\begin{array}{l}1,43 \\
1,24 \\
\text { Ref } \\
1,12 \\
1,10 \\
\end{array}$ & $\begin{array}{r}1,15 \bullet \\
1,19 \bullet \\
\text { Ref } \\
1, \mathrm{E}+79 \\
0,77 \bullet \\
\end{array}$ & \begin{tabular}{|cc}
63,53 & $\bullet$ \\
0,36 & $\bullet$ \\
Ref & \\
$4, E+10$ & $\bullet$ \\
0,34 & $\bullet$ \\
\end{tabular} & $\begin{array}{l}0,55 \\
1,10 \bullet \\
\text { Ref } \\
1,08 \bullet \\
5,78 \\
\end{array}$ & $\begin{array}{l}0,10 \\
1,64 \\
\text { Ref } \\
0,33 \\
1,28 \\
\end{array}$ & $\begin{array}{l}3,64 \\
2,42 \\
\text { Ref } \\
0,56 \\
0,95 \\
\end{array}$ & $\begin{array}{l}4,21 \\
1,33 \\
\text { Ref } \\
0,40 \\
0,62 \\
\end{array}$ & & & \\
\hline
\end{tabular}


Annexe 2 - Modèle probaliste des trajectoires résidentielles 1996-2001 pour l'agglomération de Bruxelles

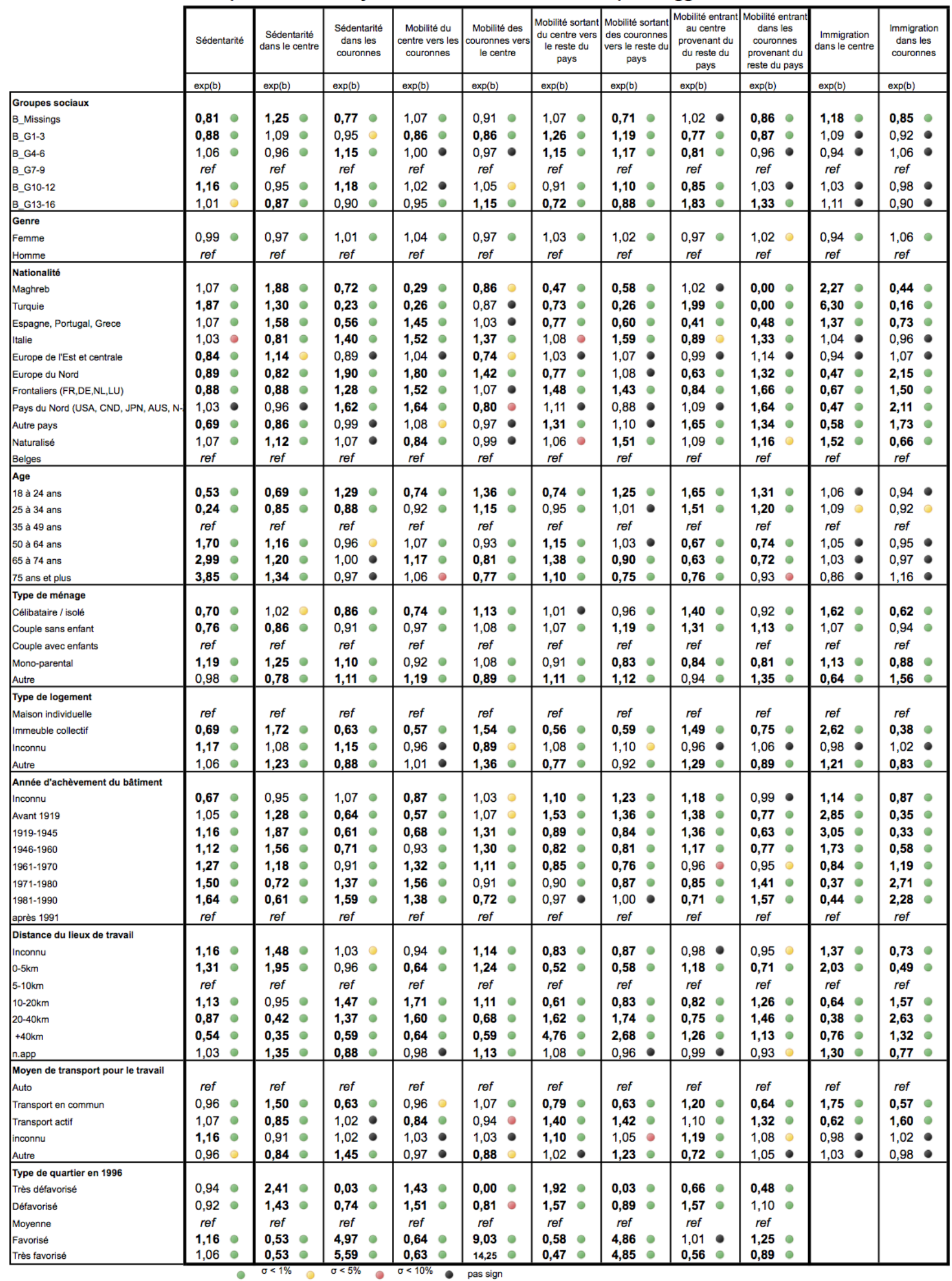

